Canadian

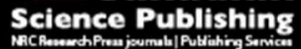

Canadian Journal of Forest Research Revue canadienne de recherche forestière

\title{
The challenge of estimating a residual spatial autocorrelation from forest inventory data
}

\begin{tabular}{|r|l|}
\hline Journal: & Canadian Journal of Forest Research \\
\hline Manuscript ID & cjfr-2017-0247.R1 \\
\hline Manuscript Type: & Article \\
\hline Complete List of Authors: & $\begin{array}{l}\text { Magnussen, Steen; Natural Resources Canada, Canadian Forest Service } \\
\text { Breidenbach, Dr. Johannes; Norsk Institutt for Skog og Landskap, } \\
\text { Mauro Gutiérrez, Francisco; Oregon State University, College of foretsry. } \\
\text { Forest Engineering Resources and Management. }\end{array}$ \\
\hline Keyword: & $\begin{array}{l}\text { first-order autoregression, restricted maximum likelihood, simulation, } \\
\text { stand effects, synthetic estimates }\end{array}$ \\
\hline $\begin{array}{r}\text { Is the invited manuscript for } \\
\text { consideration in a Special } \\
\text { Issue? : }\end{array}$ & N/A \\
\hline &
\end{tabular}


1 The challenge of estimating a residual spatial autocorrelation from forest 2 inventory data

by

Steen Magnussen ${ }^{1}$, Johannes Breidenbach ${ }^{2}$, Fransisco Mauro $^{3}$

3. Oregon State University. College of Forestry, Forest Engineering Resources and

1. Natural Resources Canada, Canadian Forest Service, 506 Burnside Rd. West, Victoria BC V8Z 1M5, Canada.Email: steen.magnussen@canada.ca (corresponding author)

2. Norwegian Institute of Bioeconomy Research, National Forest Inventory, P.O.Box 115, NO-1431As, Norway. Management Department.053 Peavy Hall, Corvallis, OR 97331, USA

12

13

14 


\section{Abstract}

16 Estimates of stand averages are needed by forest management for planning purposes. In

17 forest enterprise inventories supported by remotely sensed auxiliary data, these estimates are

18 typically derived exclusively from a model that does not consider stand effects in the study

19 variable. Variance estimators for these means may seriously underestimate uncertainty, and

20 confidence intervals may be too narrow when a model used for computing a stand mean

21 omits a non-trivial stand-effect in one or more of the model-parameters, a non-trivial spatial

22 distance dependent autocorrelation in the model residuals, or both. In simulated sampling

23 from 36 populations with stands of different sizes, and differing with respect to: $i$ ) the

24 correlation between a study variable $(Y)$ and two auxiliary variables $(\mathbf{X})$; $i i)$ the magnitude of

25 stand effects in the intercept of a linear population model linking $\mathbf{X}$ to $Y$; and iii) a first-order

26 autoregression in $Y$ and $\mathbf{X}$ - we learned that none of the tested designs provided reliable

27 estimates of the within-stand autocorrelation among model residuals. More reliable estimates

28 were possible from stand-wide predictions of $Y$. The anticipated bias in an estimated

29 autoregression parameter had a modest influence on estimates of variance and coverage of

30 nominal $95 \%$ confidence intervals for a synthetic stand mean.

31 Keywords: first-order autoregression, restricted maximum likelihood, simulation, stand

32 effects, synthetic estimates.

\section{Introduction}

34 Forest management inventories play a key role in support of sustainable forestry practices

35 (Hickey et al. 2007; Varma et al. 2000). Contemporary forest management inventories

36 frequently employ airborne laser scanners to deliver a census of vegetation surface metrics

37 (X) correlated with attributes of interest $(Y)$ at a spatial resolution commensurate with a forest

38 inventory field plot (Latifi et al. 2015; Næsset 2004b; Wulder et al. 2013). From a probability

39 sample of $n$ field plots, the parameters in one or more models linking $\mathbf{X}$ to $Y$ are estimated, 
40 and the model(s) are then used to obtain either model-assisted (Opsomer et al. 2007) or

41 model-based (Gregoire 1998) population-level or strata estimates of the means and variances

42 of $Y$.

43 In the context of a forest management inventory, it has become routine to re-use the models

44 for a stand-level estimation of means, variances, and confidence intervals (Næsset 2004a).

45 Since field sampling is expensive, and models have, in some cases, lowered the required

46 sample size for meeting a precision target for population- or stratum-level mean, the $n$

47 samples rarely constitutes more than a tiny fraction of the sampling frame (Dymond et al.

48 2006; Hawbaker et al. 2009; Junttila et al. 2013; Parker and Evans 2004). As a consequence

49 one should expect a vast majority of stand-level estimates to be synthetic (Rao and Molina

50 2015, ch. 3.2). The properties (bias and accuracy) of stand-level synthetic estimators depend

51 strongly on the correctness -across all stands - of: the employed model; all assumptions

52 regarding model errors; covariance structures; and the distribution of model residuals

53 (Schreuder et al. 1993, ch. 8.1.1).

54 In forest management inventories one should expect, even in a stratum, some level of stand

55 effects in $Y$ and $\mathbf{X}$ (Chen and Klinka 2003; Král et al. 2014), and possibly also in model

56 residuals (Breidenbach et al. 2008; Goerndt et al. 2011; Maltamo et al. 2012; Mauro et al.

57 2016). Also, a within-stand spatial distance-dependent autocorrelation (at the scale of a field

58 plot) in $Y, \mathbf{X}$, and possibly model residuals should not be ruled out (Breidenbach et al. 2015;

59 Finley et al. 2008; Lappi 2001; Magnussen et al. 2016b; Ver Hoef and Temesgen 2013). If

60 both stand effects and autocorrelation of residuals exist, but omitted in an analysis, the

61 variance of a (synthetic) stand mean would underestimate uncertainty (Fortin et al. 2016;

62 Mandallaz 2008, (6.7) p. 101). It is therefore important that a forest management inventory

63 design affords estimators for these effects by ensuring: a sufficient number of stands with 
64 more than one sampled element (Grafström and Tillé 2013); and a spatial balance of sampled 65 stands (Grafström et al. 2014).

66 A recent simulation study suggested that a probability sampling with at least two samples

67 collected in a relatively small number of stands affords a reliable estimator of random stand

68 effects in the intercept of a linear model (Magnussen and Breidenbach 2017), which, in turn,

69 greatly improved estimates of variance and $95 \%$ confidence intervals for synthetic stand

70 means.

71 However, the study did not address the issue of a distance dependent autocorrelation in model

72 residual within a stand. Given the variance inflating potential of even a relatively weak

73 spatial distance dependent autocorrelation among within-stand model residuals (Fortin et al.

74 2012; Legendre 1993; Magnussen et al. 2016a; Smith 1938), its estimation justifies a directed

75 effort (Breidenbach et al. 2015; McRoberts et al. 2011; Nieschulze and Saborowski 2002).

76 For the estimation of the parameters in a spatial distance dependent autocorrelation process

77 one would ideally collect data by a design optimized for this purpose (de Gruijter 2002; Di

78 Zio et al. 2004; Falk et al. 2011; Stein and Ettema 2003).Typically these designs ensures a

79 balance in spatial distances among pairs of observations. Forest inventory designs are rarely

80 designed with consideration given to estimation of spatial processes.

81 In this study we demonstrate the challenge of bias in estimators of the parameter of a simple

82 first-order autoregressive process in model residuals with data from a forest enterprise

83 inventory design employing conventional circular fixed area primary selection units (PSUs

84 viz. plots viz. elements). The sampling designs considered here allow an estimation of stand

85 effects by random sampling $n_{2}=\{2,4,6\}$ PSUs in each of $n_{1}$ stands selected with probability

86 proportional to size. 
87 The demonstration is carried with simulated sampling from 36 fixed artificial tri-variate

88 populations with a linear model linking $Y$ to $\mathbf{X}$ (Magnussen and Breidenbach 2017) and a

89 spatial first-order autoregressive process (at the scale of a field plot) among values of $\mathbf{X}$ and

$90 Y$. The levels of the distance dependent autocorrelation were intended to cover the range

91 anticipated for managed forests stands.

\section{Material and methods}

93 The simulation study, models, estimators, and performance indicators described in the

94 following sections contains a large number of symbols for parameters, variables, and

95 statistics. To assist readers, an alphabetically sorted list with definitions is provided in

96 Table 1.

97 Sampling and inferential objectives

98 A probability sample of size $n$ elements is taken (without replacement) from a finite (fixed)

99 forest population composed of $N$ elements ( $n$ is an order of magnitude less than $N$ ) and $M$

100 stands for the purpose of a design-based population-level inference and model-based

101 estimation of the average of $Y$ in each stand. A variance of an expected stand mean, and a

$10295 \%$ confidence interval is also desired. The model-based paradigm (Gregoire 1998) is

103 adopted for stand-level inference because the number of elements to be sampled in most

104 stands is too low to afford more than a synthetic estimator (Rao and Molina 2015, ch. 3.2).

105 For our demonstration, we conveniently assume that an analyst adopts the actual model for a 106 population:

$$
y_{i j}=\boldsymbol{\beta}^{t} \mathbf{X}_{i j}+e_{i}+e_{i j}, i=1, \ldots, M, j=1, \ldots, m_{i}
$$

108 where $y_{i j}$ is the value of $Y$ in the $j$ th element in the $i$ th stand; $\mathbf{X}_{i j}=\left\{1, x_{1 i j}, x_{2 i j}\right\}$ is a length 3

109 column vector of an intercept, and values of $X_{1}$ and $X_{2}$ in the $j$ th element in the $i$ th stand; $\boldsymbol{\beta}$ is

110 a length 3 column-vector of regression coefficients to be estimated from the sample; $e_{i}$ is a 
111 stand-specific intercept; and $e_{i j}$ is an element specific error term. A superscript ' $t$ ' in (1) is

112 used for the transpose of a vector. Note, $e_{i}$ is shared by all elements in a stand, which gives

113 rise to a constant (distance independent) covariance among all pairs of elements in a stand. In

114 contrast, $e_{i j}$ is associated with a specific element within a stand. The stand (domain) terms

$115 e_{i}, i=1, \ldots . M$ are, for the variable of interest, assumed independent and normally distributed

116 with a mean of zero and a variance $\sigma_{s t}^{2}$ equal to the covariance between any pair of elements

$117 i j$ and $i j^{\prime}\left(j \neq j^{\prime}\right)$ within a stand (Casella and Berger 2002, ch. 11.2.6). The within-stand

118 specific terms $e_{i j}, j=1, \ldots ., m_{i}$ are assumed normally distributed with a mean of zero, a

119 variance of $\sigma_{w}^{2}$ and correlated through an assumed stationary isotropic and distance

120 dependent first-order autoregressive spatial process in $\mathbf{X}$ and $Y$ with parameter $\alpha_{e}$ (see

121 below).

122 By adopting the actual model for the relationship between $\mathbf{X}$ and $Y$ and the covariance

123 structure of model residuals within a stand, we have optimized the conditions for obtaining

124 the actual values of the model parameters. Challenges encountered in this ideal setting will

125 only increase in practice when the true model remains unknown, and the spatial process in

126 model residuals have to be deduced from empirical residuals (Kim and Boos 2004). Hence,

127 complex issues regarding the effects of variable and model selection are outside the scope of

128 our work.

129 According to the model in (1), a model-based estimate of the stand mean becomes (Chambers

130 and Clark 2012, ch. 7.1)

131

$$
\hat{y}_{i}=m_{i}^{-1} \sum_{j=1}^{m_{i}} \hat{\boldsymbol{\beta}}^{t} \mathbf{X}_{i j}+\sum_{i j \in s_{i}}\left(y_{i j}-\hat{y}_{i j}\right)
$$


132 where $\hat{\boldsymbol{\beta}}$ is an empirical best linear unbiased predictor (EBLUP, Fahrmeir et al. 2013, ch.

133 7.3) of $\boldsymbol{\beta}$ in the mixed linear model (1), $\hat{y}_{i j}$ is the prediction of $y_{i j}$ given $\mathbf{X}_{i j}$ and $\hat{\boldsymbol{\beta}}$, and the

134 summation is over sampled elements in the $i$ th stand $\left(s_{\mathrm{i}}\right)$. For most stands, the second term is

1350 as no stand element has been sampled. In our approach we have dropped the second term in

136 order to mimic synthetic estimators which are applicable to the vast majority of stands in

137 actual applications.

138 Formally the model-based estimator of the variance of $\hat{y}_{i}$ in (2) becomes (Chambers and

139 Clark 2012, eq. 7.2)

140

$\hat{V}\left(\hat{y}_{i}\right)=\overline{\mathbf{X}}_{i} \hat{\Omega} \overline{\mathbf{X}}_{i}^{t}+\hat{\sigma}_{s t}^{2}+\left(m_{i}-n_{i j \in s_{i}}\right)^{-1} \hat{\sigma}_{w}^{2} \sum_{j=1}^{m_{i}-n_{i j \in s_{i}}} \sum_{j^{\prime}=1}^{m_{i}-n_{i j s_{i}}} \hat{\alpha}_{e}^{\text {rnd }\left(d i s t\left(i j, i j^{\prime}\right)\right)}$

141 where $\hat{\Omega}$ is the EBLUP estimate of the $3 \times 3$ covariance matrix of the parameters $\boldsymbol{\beta} ; \hat{\sigma}_{s t}^{2}, \hat{\sigma}_{w}^{2}$,

142 and $\hat{\alpha}_{e}$ are restricted maximum likelihood (REML) estimators (Fahrmeir et al. 2013, ch.

143 7.3.2) of $\sigma_{s t}^{2}, \sigma_{w}^{2}$, and $\alpha_{e}$ respectively, $n_{i j \in s_{i}}$ is the number of elements sampled in the $i$ th

144 stand, $\hat{\alpha}_{e}$ is the REML estimator of the first-order residual spatial autoregression parameter,

145 and $r n d\left(\operatorname{dist}\left(i j, i j^{\prime}\right)\right)$ is the Euclidean distance between elements $i j$ and $i j^{\prime}$ in the $i$ th stand

146 rounded to the nearest integer. In (3) any $\alpha_{e} \geq 0$ raised to the power of 0 (i.e. a distance of

147 zero) is, by definition, equal to 1 . As done for the mean in (1) we have set the sample size in

148 all stands to 0 in our calculations of variances. An alternative (improved) estimate of $\alpha_{e}$ was

149 derived directly from all elements $\hat{y}_{i j}$ in the $n_{1}$ selected stands. Computing time issues

150 dissuaded us from using all $M$ stands for this estimation (see Appendix for details).

151 Note the variance estimator in (3) is valid only if the estimated population-level model is

152 equal to both the true model for the population and for each stand in question. Otherwise it 
153 underestimates the uncertainty by omitting model bias (the expected value of $\hat{\boldsymbol{\beta}}-\boldsymbol{\beta}$ over all

154 possible samples in a finite population generated by a given probability design).

155 The desired nominal $95 \%$ confidence interval for a stand mean $\bar{y}_{i}$ was computed - based on

156 normal-theory and application of the central-limit theorem (Fuller 2009, ch. 1.3.2) - as

$$
C I 95_{\bar{y}_{i}}=\left\{\hat{y}_{i}-z_{0.025} \sqrt{\hat{V}\left(\hat{y}_{i}\right)}, \hat{y}_{i}+z_{0.975} \sqrt{\hat{V}\left(\hat{y}_{i}\right)}\right\}
$$

158 where $z_{q}$ is the $q$ th percentile in the distribution function of a standard normal variate.

159 To compute the variance in (3) and the confidence interval in (4) an analyst requires a

160 sampling design that affords a design-consistent (the error in a parameters goes to zero as the

161 sample size increases, Mandallaz 2008, p. 6) and efficient estimation of $\hat{\Omega}, \hat{\sigma}_{s t}^{2}, \hat{\sigma}_{w}^{2}$, and $\hat{\alpha}_{e}$.

162 Estimating two variance components and a first-order autoregression coefficient from a set of

163 empirical residuals raise the sceptre of inconsistent results (Zhang 2004) due to the fact that

164 for a fixed total within-stand covariance, say $T_{c o v}$, the likelihood function can have multiple

165 local maxima with very similar likelihoods since the equation

$166 T_{c o v}=m_{i} \sigma_{s t}^{2}+\sigma_{w}^{2} \sum_{j=1}^{m_{i}} \sum_{j^{\prime}=1}^{m_{i}} \alpha_{e}^{r n d\left(d i s t\left(i j, i j^{\prime}\right)\right)}$ can have more than one solution in terms of

$167 \sigma_{s t}^{2}, \sigma_{w}^{2}$, and $\alpha_{e}$ with practically equal likelihoods. The possible confounding may, however,

168 be unimportant in the current context as long as the estimated variances are, on average, good

169 approximations to their empirical counterparts in replicated sampling, and computed

170 confidence intervals achieve a coverage (proportion of true means contained in a computed

171 interval) close to the nominal target.

172 Sampling designs 
173 For the purpose of estimating the parameters needed for an estimation of the $M$ stand means

174 in (2), their associated variances in (3), and associated confidence intervals in (4), a

175 probability sampling with an overall sample size $(n)$ of $120,240,360$, and 480 and three

176 within-stand sample sizes $\left(n_{2}\right)$ of 2,4 , and 6 , was simulated and replicated 200 times for each

177 of the 36 populations. A within-stand sample size of 2 in a select number of stands is, of

178 course, the minimum feasible for the stated estimation objectives.

179 In each of the 12 design combinations of $n$ and $n_{2}$, a total of $n_{1}=n \times n_{2}^{-1}$ stands were selected

180 with probability proportional to their size $m_{i}$. The within-stand sampling of $n_{2}$ elements was

181 random without replacement. For the single purpose of estimating population-level

182 parameters, a within-stand-sampling scheme that penalizes a selection of near-neighbour

183 elements (Grafström et al. 2014; Grafström et al. 2012) would normally be preferred.

184 However, such schemes would be detrimental to the estimation of the parameters in a

185 distance dependent autocorrelation process in the model residuals $e_{i j}$ (Nordman and Caragea

186 2008).

187 With our designs and sample sizes, 200 replications aimed at providing an average Monte

188 Carlo error (the standard deviation of an estimator across replications of the simulation

189 design, Koehler et al. 2009) of approximately $1 \%$ on the average (across stands) of $\sqrt{\hat{V}\left(\hat{y}_{i}\right)}$

190 and approximately $0.5 \%$ on the average coverage of a computed $95 \%$ confidence interval. In

191 the forerunner to this study (Magnussen and Breidenbach 2017) we had 1200 replications and

192 twice as many stands in a population. However, the simulations of a spatial autocorrelation

193 process in $Y$ and $\mathbf{X}$ meant that running this many replications across twice as many stands

194 would have incurred impractical computing times when using an advanced workstation.

195 Performance assessment 
196 After each completed sample, a mean, a variance, and a 95\% confidence interval was

197 computed for each of the 800 stands and 200 replications as per (2), (3), and (4), with

198 REML estimates of $\boldsymbol{\beta}, \sigma_{s t}^{2}, \sigma_{w}^{2}$, and $\alpha_{e}$. As mentioned above, the latter was also estimated

199 directly from $\hat{y}_{i j}$ within the $n_{1}$ selected stands (Magnussen et al. 2016a).

200 We compare estimated averages (across stands and replicates) of

$\hat{\sigma}_{s t}^{2}, \hat{\sigma}_{w}^{2}, \hat{\alpha}_{e}, \hat{V}(\hat{y})$, and $C \hat{I} 95_{\bar{y}}$ to their known counterparts and explore the statistical

202 significance of main effects and two-way interactions of sample designs and population

203 parameters with weighted analyses of variance on cell means (over replications) (Searle et al.

204 1992, ch. 4.4) and/or weighted linear regressions (Draper and Smith 2014, p. 108). The

205 weight of a design entry was the inverse to the empirical variance of the estimates across the

206200 replications.

207 In practical forest enterprise inventories with dual inferential objectives (population and

208 stands) an analyst must balance the advantages of a spatial balance in the sample (Grafström

209 and Tillé 2013) against a need to ascertain spatial effects of consequence for stand-level

210 estimators (Fortin et al. 2012). Typically, a balance has to be struck within the constraints of

211 costs. We therefore focus our performance assessment on the apparent trade-offs between an

212 increase in $n_{2}$ at the expense of a decrease in $n_{1}$ when the overall sample size is kept constant.

213 Populations

214 We consider 36 tri-variate standard (mean zero, variance 1.0) Gaussian populations (forests

215 or strata) each composed of $N$ fixed area elements (the frame) subdivided into $M=800$

216 spatially compact (square) domains (forest stands) with $5^{2}, 6^{2}, \ldots$, or $14^{2}$ elements. An element

217 is a square fixed area the size of a forest inventory PSU viz. sample plot (typically between

218200 and $800 \mathrm{~m}^{2}$ ). A study variable $Y$, and two auxiliary variables $X_{1}$ and $X_{2}$ correlated with $Y$,

219 are associated with each element. The values of $X_{1}$ and $X_{2}$ are known for each element in a 
220 population while the value of $Y$ is only observed for elements collected in a probability

221 sample. The triplet $\left(y_{i j}, x_{1 i j}, x_{2 i j}\right)$ denotes the values associated with the $j$ th element $(j=1, \ldots$,

$\left.222 m_{\mathrm{i}}\right)$ in the $i$ th stand $(i=1, \ldots, M)$. Stand means in the populations are denoted $\left(\bar{y}_{i}, \bar{x}_{1 i}, \bar{x}_{2 i}\right)$.

223 The populations differentiate on: $i)$ the correlation between $Y$ and $X_{1}\left(\rho\left(y, x_{1}\right)\right)$, ii) the

224 among-stand variance in $Y\left(\sigma_{\bar{y}_{s t}}^{2}\right)$, and iii) a distance-dependent first-order spatial

225 autoregression in $Y$ along rows and columns within a stand characterized by the coefficient

$226 \alpha_{y}$ controlling the correlation between two elements sharing a border across a row or column

227 of elements. We generated populations with three levels of both $\rho^{2}\left(y, x_{1}\right)$ and $\sigma_{\bar{y}_{s t}}^{2}$, and four

228 levels of $\alpha_{y}$. The population characteristics were crossed to all possible combinations;

229 yielding $36(3 \times 3 \times 4=36)$ 'fixed' populations to be sampled repeatedly. Assigned values and

230 ranges of the parameters in this study were designed to be representative of populations of

231 even-aged mono-specific forest stands in the temperate zone. The levels of $\rho^{2}\left(y, x_{1}\right)$ were

$2320.6,0.7$, and 0.8 . Levels of $\sigma_{\bar{y}_{s t}}^{2}$ were $0.05,0.10$, and 0.20 (i.e. the among-stand variance in $Y$

233 accounts for $5 \%, 10 \%$, and $20 \%$ of the total variance of 1.0 in $Y$ ). Finally, the levels of $\alpha_{y}$

234 were $0.00,0.10,0.20$, and 0.30 , which for an average stand with 90 elements, inflates a

235 within-stand residual error variance by a factor of, 1.0, 1.8, 2.8, and 4.3 times, respectively

236 (Magnussen 2001). The covariogram for elements in a stand separated by a distance dist is

$237 C_{y}($ dist $)=\alpha_{y}^{\text {dist }}\left(1-\alpha_{y}^{2}\right)^{-1}\left(1-\sigma_{\bar{y}_{s t}}^{2}\right)\left(\right.$ Cressie 1993, p. 200). The correlation between $Y$ and $X_{2}$

238 was fixed at $0.25 \rho\left(y, x_{1}\right)$ while the correlation between $X_{1}$ and $X_{2}$ was fixed at 0.5 . The

239 among-stand (domain) variance in $X_{1}$ and $X_{2}$ was fixed at $0.7 \sigma_{\bar{y}_{s t}}^{2}$. A first-order spatial

240 autoregression coefficient of $0.7 \alpha_{y}$ was also imposed on $X_{1}$ and $X_{2}$. 
241 According to our control of the tri-variate correlations, a population census would generate

242 an estimate of $\hat{\boldsymbol{\beta}}_{\text {census }}=\{0.000,0.904,-0.258\}$ for $\rho^{2}\left(y, x_{1}\right)=0.6$,

$243 \hat{\boldsymbol{\beta}}_{\text {census }}=\{0.000,0.976,-0.279\}$ for $\rho^{2}\left(y, x_{1}\right)=0.7$, and $\hat{\boldsymbol{\beta}}_{\text {census }}=\{0.000,1.044,-0.298\}$ for

$244 \quad \rho^{2}\left(y, x_{1}\right)=0.8$.

245 Additional technical details of the simulations are in the Appendix.

\section{Results}

247 Estimates of the within-stand residual variance $\sigma_{w}^{2}$

248 Estimated values of $\hat{\sigma}_{w}^{2}$ closely tracked, as apparent in Figure 1, their nominal values $\sigma_{w}^{2}$ (see

249 Appendix). A linear regression of $\hat{\sigma}_{w}^{2}$ on $\sigma_{w}^{2}$ achieved a slope of 0.996 and an intercept of

250 0.0119. With 200 replications of a design setting, the Monte Carlo error of an estimate of $\sigma_{w}^{2}$

251 varied from $0.4 \%$ and $2.3 \%$ with a median of $0.9 \%$. The error decreased with decreasing

252 values of $\sigma_{w}^{2}, n$, and $n_{2}$, but increased with rising levels of $\alpha_{y}$. As expected (Anderson 1976,

253 p.14), $\alpha_{y}$ had a strong influence on the estimate of $\sigma_{w}^{2}$

$254\left(\alpha_{y}, \hat{F}_{3,362}=425.0, P\left(\hat{F}_{3,362} \mid H_{0}\right)<0.01\right)$. With $\alpha_{y}=0.0$ (no autocorrelation) the apparent

255 bias in $\hat{\sigma}_{w}^{2}$ was $2.8 \%, 7.6 \%$ when $\alpha_{y}=0.1$, and 8.4 when $\alpha_{y}=0.3$. The inflation is attributed

256 to the fact that the estimated values are estimates of the process variance in $e_{i j}$, which, is

257 greater than the nominal variance applicable to independent observations.

258 From a practical perspective the most important result is that there was no important main

259 effect of $n$ or a significant two-way interactions between $n$ and $\alpha_{y}$

$260\left(\hat{F}_{9,362}=0.43, P\left(\hat{F}_{9,362} \mid H_{0}\right)=0.92\right)$. The within-stand sample size $n_{2}$ was statistically

261 significant $\left(\hat{F}_{2,362}=20.5, P\left(\hat{F}_{2,362} \mid H_{0}\right)<0.01\right)$ but the influence on $\hat{\sigma}_{w}^{2}$ was a one percentage 
262 increase in the apparent bias of $\hat{\sigma}_{w}^{2}$ as $n_{2}$ increased from 2 to 6 . Interactions between $n_{2}$,

$263 \rho^{2}\left(y, x_{1}\right)$, and $\hat{\sigma}_{\bar{y}}^{2}$ were also statistically significant but an order of magnitude smaller than

264 any two-way interaction, and two-orders of magnitude smaller than the main effects of $n_{2}$ and $265 \rho^{2}\left(y, x_{1}\right)$.

266 Estimates of the among-stand residual variance $\sigma_{s t}^{2}$

267 Monte Carlo errors in estimates of $\sigma_{s t}^{2}$ varied from 1.8 to $8.8 \%$ with a median of $4.4 \%$ in 268 settings with a non-zero autocorrelation. In combination of low values of $\sigma_{s t}^{2} n$, and $n_{2}$, the 269 estimated values of $\sigma_{s t}^{2}$ were significantly inflated. Generally, as $\sigma_{s t}^{2}$ increases, the estimates 270 clustered tightly around the actual value. These trends are clearly visible in Figure 2. The 271 apparent bias in $\hat{\sigma}_{s t}^{2}$ was significantly influenced by $n\left(\hat{F}_{3,362}=5.9, P\left(\hat{F}_{3,362} \mid H_{0}\right)<0.01\right), n_{2}$

$\left(\hat{F}_{2,362}=153.2, P\left(\hat{F}_{2,362} \mid H_{0}\right)<0.01\right)$, and $\alpha_{y}\left(\hat{F}_{2,362}=3.5, P\left(\hat{F}_{2,362} \mid H_{0}\right)=0.03\right)$, and interactions $n_{2} \times \sigma_{\bar{y}_{s t}}^{2}\left(\hat{F}_{4,362}=109.4, P\left(\hat{F}_{4,362} \mid H_{0}\right)<0.01\right)$ and $n_{2} \times \rho^{2}\left(y, x_{1}\right)$ $\left(\hat{F}_{4,362}=262.5, P\left(\hat{F}_{4,362} \mid H_{0}\right)<0.01\right)$. In general terms, the apparent bias dropped from $65 \%$

275 to $15 \%$ with an increase in $n_{2}$ from 2 to 4 , from $73 \%$ to $10 \%$ with an increase in $\sigma_{\bar{s}_{s t}}^{2}$ from 0.05 to 0.20 , and from $102 \%$ to $4 \%$ as $\rho^{2}\left(y, x_{1}\right)$ drops from 0.8 to 0.6 .

Estimates of the AR1 coefficient $\alpha_{e}$

278 The introduction of a first-order autoregressive spatial process in $Y$ and $\mathbf{X}$ induced a distance dependent autocorrelation of the same magnitude in the true model residuals $\left(e_{i j}\right)$ within a stand. In our simulations - and in agreement with theory (see Appendix) - the population

281 level mean (across stands and replications) maximum likelihood estimate $\hat{\alpha}_{e}\left(e_{i j}\right)$ of $\alpha_{e}$ in 
282 the true residuals was, with the exception for $\alpha_{e}=0$ very close to $\alpha_{y}$. To wit: $\hat{\alpha}_{e}\left(e_{i j}\right)$ was

283

284

$0.036 \pm 0.004$ for $\alpha_{y}=0.0 ; 0.105 \pm 0.007$ for $\alpha_{y}=0.1 ; 0.196 \pm 0.008$ for $\alpha_{y}=0.2 ;$ and

$0.293 \pm 0.008$ for $\alpha_{y}=0.3$. Hence, only at $\alpha_{y}=0.0$ was there an apparent discrepancy between $\hat{\alpha}_{e}\left(e_{i j}\right)$ and $\alpha_{y}$. Because $\alpha_{y}=0$ is at the boundary of the parameter space for the first-order autoregression parameter, no test of the significance of the observed discrepancy was attempted.

In contrast, REML estimates $\hat{\alpha}_{e}\left(e_{i j} \in s\right)$ of $\alpha_{e}$-when data were limited to the true residuals in the sample $(s)$ - were significantly overestimated when $\alpha_{y}=0$ and increasingly underestimated as $\alpha_{y}$ increases from 0.1 to 0.3 (Figure 3). All design effects and most twoway interactions were statistically significant. In general, the apparent negative bias in $\hat{\alpha}_{e}\left(e_{i j} \in S\right)$ increased with $n, n_{2}$, and $\alpha_{y}$. These results suggest that estimates obtained with empirical (observed) residuals $\left(y_{i j}-\hat{y}_{i j}\right)$ would be worse since $\hat{\boldsymbol{\beta}}_{\text {sample }} \neq \boldsymbol{\beta}_{\text {census }}$. This was overwhelmingly confirmed, as can be taken from Figure 4 where estimates $\hat{\alpha}_{e}\left(\hat{e}_{i j}\right)$ of $\alpha_{e}$ obtained from empirical residuals are plotted against estimates obtained with true residuals $\left(e_{i j}\right)$. Clearly, a sample-based estimate of $\alpha_{e}$ based on empirical residuals bears no relationship to an estimate obtained from actual (true) residuals or to the actual $\alpha_{e}$. MonteCarlo errors in $\hat{\alpha}_{e}\left(\hat{e}_{i j}\right)$ varied from 1.0 to $15.4 \%$ with a median of $1.3 \%$.

As expected from theory (see Appendix) and illustrated in Figure 5, vastly improved estimates of $\alpha_{e}$ could be obtained from the average maximum likelihood estimate of the firstorder autoregression coefficient $\alpha_{\hat{y}}$ in $\hat{y}_{i j}$ using all elements in the $n_{l}$ sampled stands for estimation (a restriction to $n_{1}$ was chosen in order to limit otherwise excessive computing 
303 times). An overestimation of approximately 0.3 at $\alpha_{y}=0$ is apparent, and as $\alpha_{y}$ increases

304 the overestimation turns into a slight underestimation. Although the effects of $n, n_{2}$,

$305 \rho\left(y, x_{1}\right)$, and $\sigma_{\bar{y}_{s t}}^{2}$ were statistically significant $(P<0.01)$, their marginal effects were minor

$306(<1 \%)$ and without a linear trend. In the following we use these estimates $\left(\hat{\alpha}_{\hat{y}}\right)$ of $\alpha_{e}$ to

307 compute the variance and confidence interval for a synthetic stand mean.

308 Estimates of variance of a synthetic stand mean

309 The mean (across stands and replications) estimate of the variance of a synthetic stand mean

$310 \hat{V}(\hat{y})$ tracked - when using $\hat{\alpha}_{\hat{y}}$ as the estimate of the first-order autocorrelation in within-

311 stand residual errors - the actual $\operatorname{MSE}(\hat{y})$ quite well $\left(R^{2}=0.96\right)$ but with an increasing

312 apparent (negative) bias in design settings with an $\operatorname{MSE}(\hat{y})>0.035$ (Figure 6). Monte Carlo

313 errors in estimates of the stand-average $\sqrt{\hat{V}(\hat{y})}$ varied from 0.05 to $0.63 \%$ with a median of

$3140.14 \%$.

315 There was no interpretable trend in the difference between $\operatorname{MSE}(\hat{y})$ and $\hat{V}(\hat{y})$ across levels

316 of $\sigma_{\bar{y}_{s t}}^{2}$, but the apparent bias changed from $+5 \%$ to $-7 \%$ as $n$ increased from 120 to 480 , and

317 from $+8 \%$ to $-7 \%$ when $n_{2}$ increased from 2 to 6 . As $\alpha_{y}$ increased from 0 to 0.3 the apparent

318 bias changed from $-8 \%$ to $+8 \%$. None of these trends in variance estimate can be viewed as

319 important in light of the uncertainty in the estimates themselves and the associated Monte-

320 Carlo errors.

321 Although REML sample-based estimates of $\alpha_{e}$ from empirical residuals were deemed of

322 little value as estimates of the actual first-order autoregression coefficient, the ensuing

323 estimates of variances of a synthetic stand mean was surprising close to those detailed above

324 (Figure 7). In general, the two sets of estimates were strongly correlated $\left(R^{2}=0.97\right)$ and the 
325 average difference was approximately $4 \%$ across most settings. However, estimates obtained

326 with $\hat{\alpha}_{\hat{e}}$ were considerably less precise than estimates obtained with $\hat{\alpha}_{\hat{y}}$. To wit, their among-

327 replication standard deviation was approximately 4 times greater.

328 For an average stand with approximately 90 elements, the first-order autoregressive process

329 in $Y$ and $\mathbf{X}$ led to an average increase in $\hat{V}(\hat{y})$ - when using $\hat{\alpha}_{\hat{y}}$ as the estimate of the first-

330 order autocorrelation in within-stand residual errors - of $3 \%$ when $\alpha_{y}$ was $0.1,9 \%$ with

$331 \alpha_{y}=0.2$, and $20 \%$ with $\alpha_{y}=0.3$. These numbers compare reasonably with theoretical

332 expectations of $1 \%, 10 \%$, and $24 \%$. An inflation greater than expected was, in general,

333 caused by an overestimation of $\sigma_{s t}^{2}$ or $\alpha_{e}$. In contrast, with $\hat{\alpha}_{e}\left(\hat{e}_{i j}\right)$ the average inflation was

$3341.2 \%$ but with essentially no trend in the inflation across levels of $\alpha_{y}$.

335 Achieved coverage of a nominal CI95

336 Across all design settings the achieved coverage - when using $\hat{\alpha}_{\hat{y}}$ as the estimate of $\alpha_{e}$ - was

3370.95 but as indicated in Figure 8, the coverage was highest $($ mean $=0.98)$ at the lowest level

338 of the among-stand residual variance $\left(\sigma_{s t}^{2}\right)$, and it then declined gradually (mean $\left.=0.94\right)$ at

339 the highest level of $\sigma_{s t}^{2}$. In addition to $\sigma_{s t}^{2}$, the effects of $\alpha_{y}$ and $\rho\left(y, x_{1}\right)$ were also

340 statistically significant $(P<0.001)$. Coverage increased from 0.94 to 0.96 as $\alpha_{y}$ rose from 0

341 to 0.3 and from 0.94 to 0.97 as $\rho^{2}\left(y, x_{1}\right)$ increases from 0.6 to 0.8 . No other trend attracted

342 attention. Monte Carlo errors in stand-averages of coverage varied from 0.15 to $1.10 \%$ with a

343 median of $0.46 \%$.

344 Coverage computed with $\hat{\alpha}_{e}\left(\hat{e}_{i j}\right)$ was slightly inferior to the coverage with $\hat{\alpha}_{\hat{y}}$. Across all

345 settings the achieved coverage was 0.94 with $90 \%$ of the estimates between 0.90 and 0.98 . 
346 The difference between the two sets of estimates increased with the strength of $\rho\left(y, x_{1}\right)$,

347 from -1.0 at the weakest level to 0.04 at the strongest level.

\section{Discussion}

349 The focus of our discussion is on the apparent challenges in deriving restricted maximum

350 likelihood estimates of a distance dependent autocorrelation process in model residuals when

351 data comes from a forest inventory with fixed-area PSUs (plots) and supported by remotely

352 sensed auxiliary variables correlated with a study variable of interest. Practical aspects and

353 importance of estimating possible stand effects in forest enterprise inventories was discussed

354 in a forerunner to this study (Magnussen and Breidenbach 2017).

355 The potential of a spatial distance dependent autocorrelation in model residuals to inflate a

356 model-based variance of a stand mean is well established (Fortin et al. 2012; Griffith 2005;

357 Legendre 1993; Magnussen 2001) and confirmed in this study. It is therefore natural that its

358 estimation enters into the sampling and estimation strategy of a forest enterprise inventory

359 (Gregoire and Valentine 2008, ch. 1.1).

360 The demonstrated failure to obtain reliable restricted maximum likelihood estimates of a

361 single parameter in a simple first-order spatial autogressive process in model residuals should

362 not come as a surprise. Sampling intensities in enterprise forest inventories are low and,

363 everything else equal, set the stage for a poor precision of estimated parameters in a spatial

364 process (Chilès and Delfiner 1999, ch. 2.9; Cressie 1993, p. 127-135; Marchant and Lark

365 2004; Nordman and Caragea 2008). A potential confounding of model parameters linked to

366 variance components (Hodges and Reich 2010; Zhang 2004) is equally at play.

367 Our results therefore presage a poor confidence in (frequentist) estimates derived from actual

368 forest enterprise inventory data when both the chosen model linking $\mathbf{X}$ to $Y$ and the spatial

369 process in model residuals may be miss-specified vis-à-vis the actual state (Breidenbach et al. 
370 2008; Breidenbach et al. 2015; Gunnarsson et al. 1998; Mauro Gutiérrez et al. 2017;

371 McRoberts et al. 2011; Rahlf et al. 2014; Ver Hoef and Temesgen 2013). Our failure would

372 likely be exacerbated by more complex models for the spatial process (Di Zio et al. 2004;

373 Hodges and Reich 2010; Kim and Boos 2004; Zhang et al. 2005; Zimmerman 2006) and by

374 variance heteroscedasticity in the residuals (Rahlf et al. 2014). In sum, data suited for a

375 model fitting in presence of spatial heterogeneity and spatial dependence (Beale et al. 2010;

376 Falk et al. 2011; Fortin et al. 2012; Geniaux and Martinetti 2017; Jiang and Nguyen 2012;

377 Salas et al. 2010; Salvati et al. 2012) are rarely available from forest enterprise inventories. A

378 Bayesian approach may hold more promise (Babcock et al. 2015; Finley et al. 2008).

379 In forest enterprise inventories, practical and variance efficient sampling designs rarely

380 provide empirical residuals suitable for estimation of a spatial correlation process (Mauro

381 Gutiérrez et al. 2017). Fortunately, our results also suggest that even with an anticipated bias

382 in an estimate of the parameter in a spatial autocorrelation process, a reasonable estimate of

383 the variance of a synthetic stand mean and a $95 \%$ confidence interval with an anticipated

384 coverage reasonably close to its nominal target is still possible.

385 From a practical perspective, our results may encourage an analyst to refrain from attempting

386 an estimation of the autocorrelation process in model residuals. There are clearly many

387 situations, which warrant this attitude. For example, ignoring a spatial autocorrelation that

388 inflates the variance of a synthetic stand mean by at most $8 \%$ would seem permissible as it

389 only lowers the coverage of a CI95 by approximately 0.01 (a result that can be obtained from

390 the central limit theorem and the properties of a standard Gaussian distribution). As well, if

391 the relative variance of model errors plus stand-effects accounts for $10 \%$ or less of the total

392 variance in $Y$, a first-order spatial autoregression parameters of 0.06 or less can be ignored for

393 a stand composed of 25 elements without adverse effect on variance and coverage. The

394 corresponding threshold for a stand with 196 elements is 0.18 . When the relative variance of 
395 model errors and stand effects climb to $30 \%$, the aforementioned thresholds increase to 0.27

396 and 0.39 , respectively. Yet, a decision to ignore a possible spatial autocorrelation remains

397 delicate because the potential variance inflation depends not only on the relative magnitude

398 of the variances due to parameter errors, stand effects, and residuals, but also on stand size,

399 model formulation (Kangas 1994), and spatial scale (Smith and Urban 1988).

400 Instead of pursuing a REML estimation of the parameter(s) in a spatial autocorrelation

401 process in model residuals, our study suggests a computationally simpler approach by using

402 element level model predictions in place of empirical residuals. These predictions are readily

403 available for all elements of interest and not restricted by the sampling design (Kar et al.

404 2011; Zhang 2004; Zimmerman 2006). Our success obviously hinges on using the correct

405 (actual) model for fitting (Engel 1984). If the model is miss-specified, a realized

406 autocorrelation in $\hat{y}_{i j}$ for a given $\alpha_{y}$ may be inflated. To guard against a model

407 misspecification that generates an inflated estimate of $\alpha_{y}$ Magnussen et al. (2016a)

408 recommended an ad-hoc reduction of $\hat{\alpha}_{e}\left(\hat{y}_{i j}\right)$ by $60 \%$.

409 Design recommendations by Magnussen and Breidenbach (2017) for a forest enterprise

410 inventory with dual objectives of estimating a population mean (total) and synthetic stand

411 means are valid even when the impact of a spatial autocorrelation process is considered.

412 When stand effects in $Y$ are likely, an efficient design is one that combines sampling of one

413 element from a larger number of stands with sampling of two elements from a relatively

414 small number of stands (say 40).

\section{Conclusions}

416 Forest enterprise inventories with population-level and stand-level inferential objectives

417 resort to model-based estimators of a stand mean by choice or when the number of samples 
418 representing a stand of interest is too low to afford an otherwise preferred design-based

419 estimator. When an estimate of the variance of a stand mean is required, an analyst runs the

420 risk of a serious underestimation unless in possession of reliable estimates of stand effects,

421 and the strength of a possible spatial autocorrelation in the differences between model based

422 expectations and the actual value (i.e. model residuals). While reliable estimates of stand

423 effects can flow from sampling designs with as few as two samples taken in a relatively small

424 subset of stands, this study made clear, that a restricted maximum likelihood approach to the

425 estimation of a spatial autocorrelation in empirical residuals is likely to fail with practically

426 affordable sampling designs. Fortunately, our results also suggest that a proxy for the spatial

427 autocorrelation can come from model predictions without adverse effect on the coverage of

428 nominal $95 \%$ confidence intervals.

\section{Acknowledgements}

430 Comments and critique from two journal reviewers and the Associate Editor prompted many

431 improvements to our first submission. We gratefully appreciate their constructive reviews.

\section{References}

433 Anderson, O.D. 1976. Time Series Analysis and Forecasting: The Box-Jenkins approach.

434 Butterworths, London.

435 Babcock, C., Finley, A.O., Bradford, J.B., Kolka, R., Birdsey, R., and Ryan, M.G. 2015.

436 LiDAR based prediction of forest biomass using hierarchical models with spatially varying

437 coefficients. Remote Sens. Environ. 169: 113-127.

438 Beale, C.M., Lennon, J.J., Yearsley, J.M., Brewer, M.J., and Elston, D.A. 2010. Regression

439 analysis of spatial data. Ecol. Let. 13(2): 246-264.

440 Breidenbach, J., Kublin, E., McGaughey, R., Andersen, H.-E., and Reutebuch, S. 2008.

441 Mixed-Effects Models for Estimating Stand Volume By Means of Small Footprint Airbrone

442 Laser Scanner Data. Photogrammetric Journal of Finland 21(1): 4-15.

443 Breidenbach, J., McRoberts, R.E., and Astrup, R. 2015. Empirical coverage of model-based

444 variance estimators for remote sensing assisted estimation of stand-level timber volume.

445 Remote Sens. Environ. 173: 274-281. doi: 10.1016/j.rse.2015.07.026.

446 Casella, G., and Berger, R.L. 2002. Statistical Inference. 2 ed. Duxbury Press, Pacific Grove. 447 pp. 660.

448 Chambers, R.L., and Clark, R.G. 2012. An introduction to model-based survey sampling with

449 applications. Oxford University Press, New York. pp. 265. 
450 Chen, H.Y., and Klinka, K. 2003. Aboveground productivity of western hemlock and western 451 redcedar mixed-species stands in southern coastal British Columbia. For. Ecol. Manage.

452 184(1): 55-64.

453 Chilès, J.P., and Delfiner, P. 1999. Geostatistics: Modeling spatial uncertainty. Wiley, New

454 York. pp. 695.

455 Christensen, R. 2001. Linear models for multivariate, time series and spatial data. Springer, 456 New York. pp. 217.

457 Cressie, N.A.C. 1993. Statistics for spatial data. Revised edition. 2nd ed. Wiley, New York. 458 pp. 900.

459 de Gruijter, J. 2002. Spatial sampling schemes for remote sensing. In Spatial Statistics for 460 Remote Sensing. Springer. pp. 211-242.

461 Di Zio, S., Fontanella, L., and Ippoliti, L. 2004. Optimal spatial sampling schemes for 462 environmental surveys. Environ. Ecol. Stat. 11(4): 397-414.

463 Draper, N.R., and Smith, H. 2014. Applied Regression Analysis. 3 ed. Wiley, New York. pp. 464736.

465 Dymond, C.C., Wulder, M.A., Shore, T.L., Nelson, T., Boots, B., and Riel, B.G. 2006.

466 Evaluation of risk assessment of mountain pine beetle infestations. W. J. Appl. For. 21(1): 546713.

468 Engel, E. 1984. A unified approch to the study of sums, products, time-aggregation, and other 469 functions of ARMA processes. Journal of Time Series Analysis 5(3): 159-171.

470 Fahrmeir, L., Kneib, T., Lang, S., and Marx, B. 2013. Regression: models, methods and 471 applications. Springer, New York. pp. 698.

472 Falk, M., Denham, R., and Mengersen, K. 2011. Spatially stratified sampling using auxiliary 473 information for geostatistical mapping. Environ. Ecol. Stat. 18(1): 93-108. doi:

474 10.1007/s10651-009-0122-3.

475 Finley, A.O., Banerjee, S., Ek, A.R., and McRoberts, R.E. 2008. Bayesian multivariate 476 process modeling for prediction of forest attributes. J. Agric. Biol. Environ. Stat. 13(1): 60477 83. doi: 10.1198/108571108x273160.

478 Fortin, M.-J., James, P.M.A., MacKenzie, A., Melles, S.J., and Rayfield, B. 2012. Spatial 479 statistics, spatial regression, and graph theory in ecology. Spatial Statistics 1(0): 100-109. 480 doi: http://dx.doi.org/10.1016/j.spasta.2012.02.004.

481 Fortin, M., Manso, R., and Calama, R. 2016. Hybrid estimation based on mixed-effects 482 models in forest inventories. Can. J. For. Res. 46(11): 1310-1319. doi: 10.1139/cjfr-20164830298.

484 Fuller, W.A. 2009. Sampling Statistics. Wiley, New York. pp. 454.

485 Geniaux, G., and Martinetti, D. 2017. A new method for dealing simultaneously with spatial 486 autocorrelation and spatial heterogeneity in regression models. Regional Science and Urban 487 Economics.

488 Gneiting, T., Kleiber, W., and Schlather, M. 2010. Matérn Cross-Covariance Functions for 489 Multivariate Random Fields. J. Am. Stat. Assoc. 105(491): 1167-1177. doi:

$490 \quad$ 10.1198/jasa.2010.tm09420. 
491 Goerndt, M.E., Monleon, V.J., and Temesgen, H. 2011. A comparison of small-area

492 estimation techniques to estimate selected stand attributes using LiDAR-derived auxiliary

493 variables. Can. J. For. Res. 41(6): 1189-1201. doi: 10.1139/x11-033.

494 Grafström, A., Lundström, N.L., and Schelin, L. 2012. Spatially balanced sampling through

495 the pivotal method. Biometrics 68(2): 514-520.

496 Grafström, A., Saarela, S., and Ene, L.T. 2014. Efficient sampling strategies for forest

497 inventories by spreading the sample in auxiliary space. Can. J. For. Res. 44(10): 1156-1164.

498 doi: 10.1139/cjfr-2014-0202.

499 Grafström, A., and Tillé, Y. 2013. Doubly balanced spatial sampling with spreading and

500 restitution of auxiliary totals. Environmetrics 24(2): 120-131.

501 Gregoire, T.G. 1998. Design-based and model-based inference in survey sampling:

502 Appreciating the difference. Can. J. For. Res. 28(10): 1429-1447.

503 Gregoire, T.G., and Valentine, H.T. 2008. Sampling strategies for natural resources and the

504 environment. Chapman \& Hall/CRC, Boca Raton, FL. pp. 465.

505 Griffith, D.A. 2005. Effective Geographic Sample Size in the Presence of Spatial

506 Autocorrelation. Annals of the Association of American Geographers 95(4): 740-760. doi:

507 10.1111/j.1467-8306.2005.00484.x.

508 Gunnarsson, F., Holm, S., Holmgren, P., and Thuresson, T. 1998. On the potential of kriging

509 for forest management planning. Scand. J. For. Res. 13(1-4): 237-245.

510 Gupta, A.K., and Nagar, D.K. 1999. Matrix Variate Distributions. CRC. pp. 384.

511 Hawbaker, T.J., Keuler, N.S., Lesak, A.A., Gobakken, T., Contrucci, K., and Radeloff, V.C.

512 2009. Improved estimates of forest vegetation structure and biomass with a LiDAR-

513 optimized sampling design. J. Geophys. Res. 114. doi: 10.1029/2008JG000870.

514 Hickey, G.M., Innes, J.L., and Kozak, R.A. 2007. Monitoring and information reporting for

515 sustainable forest management: A regional comparison of forestry stakeholder perceptions. J.

516 Environ. Manage. 84(4): 572-585. doi: http://dx.doi.org/10.1016/j.jenvman.2006.07.004.

517 Hodges, J.S., and Reich, B.J. 2010. Adding Spatially-Correlated Errors Can Mess Up the

518 Fixed Effect You Love. Am. Stat. 64(4): 325-334. doi: doi:10.1198/tast.2010.10052.

519 Jiang, J., and Nguyen, T. 2012. Small area estimation via heteroscedastic nested-error

520 regression. Can J Statist 40(3): 588-603. doi: 10.1002/cjs.11148.

521 Johnson, C.R., Okubo, K., and Reams, R. 2001. Uniqueness of Matrix Square Roots and an

522 Application. Linear and Multilinear Algebra (323): 51-60.

523 Junttila, V., Finley, A.O., Bradford, J.B., and Kauranne, T. 2013. Strategies for minimizing

524 sample size for use in airborne LiDAR-based forest inventory. For. Ecol. Manage. 292: 75-

525 85. doi: 10.1016/j.foreco.2012.12.019.

526 Kangas, A. 1994. Classical and model based estimators for forest inventory. Silv Fenn

527 Monogr 28: 3-14.

528 Kar, S., Varshney, P.K., and Chen, H. 2011. Spatial whitening framework for distributed

529 estimation. In Computational Advances in Multi-Sensor Adaptive Processing (CAMSAP),

5302011 4th IEEE International Workshop on. IEEE. pp. 293-296.

531 Kim, H.J., and Boos, D.D. 2004. Variance estimation in spatial regression using a non-

532 parametric semivariogram based on residuals. Scand. J. Stat. 31(3): 387-401. 
533 Koehler, E., Brown, E., and Haneuse, J.-P.A. 2009. On the assessment of Monte Carlo error 534 in simulation-based statistical analyses. Am. Stat. 63(2): 155-162.

535 Král, K., Valtera, M., Janík, D., Šamonil, P., and Vrška, T. 2014. Spatial variability of 536 general stand characteristics in central European beech-dominated natural stands-effects of 537 scale. For. Ecol. Manage. 328: 353-364.

538 Lappi, J. 2001. Forest inventory of small areas combining the calibration estimator and a 539 spatial model. Can. J. For. Res. 31: 1551-1560.

540 Latifi, H., Fassnacht, F.E., Müller, J., Tharani, A., Dech, S., and Heurich, M. 2015. Forest 541 inventories by LiDAR data: A comparison of single tree segmentation and metric-based 542 methods for inventories of a heterogeneous temperate forest. International Journal of Applied 543 Earth Observation and Geoinformation 42: 162-174.

544 Legendre, P. 1993. Spatial autocorrelation: Trouble or new paradigm? Ecology 74: 16595451673.

546 Magnussen, S. 2001. Fast pre-survey computation of the mean spatial autocorrelation in large 547 plots composed of a regular array of secondary sampling units. Mathematical Modelling and 548 Scientific Computing 13(3-4): 204-217.

549 Magnussen, S., and Breidenbach, J. 2017. Model-dependent forest stand-level inference with 550 and without estimates of stand-effects. Forest. Oxf. doi:

551 https://doi.org/10.1093/forestry/cpx023.

552 Magnussen, S., Frazer, G., and Penner, M. 2016a. Alternative mean-squared error estimators 553 for synthetic estimators of domain means. J. Appl. Stat. 43(14): 1-24. doi:

$55410.1080 / 02664763.2016 .1142942$.

555 Magnussen, S., Mandallaz, D., Lanz, A., Ginzler, C., Næsset, E., and Gobakken, T. $2016 b$. 556 Scale effects in survey estimates of proportions and quantiles of per unit area attributes. For. 557 Ecol. Manage. 364: 122-129. doi: http://dx.doi.org/10.1016/j.foreco.2016.01.013.

558 Maltamo, M., Mehtätalo, L., Vauhkonen, J., and Packalén, P. 2012. Predicting and

559 calibrating tree attributes by means of airborne laser scanning and field measurements. Can.

560 J. For. Res. 42(11): 1896-1907.

561 Mandallaz, D. 2008. Sampling techniques for forest inventories. Chapman and Hall, Boca

562 Raton, Florida. pp. 251.

563 Marchant, B.P., and Lark, R.M. 2004. Estimating variogram uncertainty. Mathematical

564 Geology 36(8): 867-898.

565 Mauro, F., Molina, I., García-Abril, A., Valbuena, R., and Ayuga-Téllez, E. 2016. Remote 566 sensing estimates and measures of uncertainty for forest variables at different aggregation 567 levels. Environmetrics 27(4): 225-238. doi: 10.1002/env.2387.

568 Mauro, F., Monleon, V.J., Temesgen, H., and Ruíz, L.Á. 2017. Analysis of spatial correlation 569 in predictive models of forest variables that use LiDAR auxiliary information. Can. J. For.

570 Res. 47(6): 788-799.

571 McRoberts, R.E., Magnussen, S., Tomppo, E.O., and Chirici, G. 2011. Parametric, bootstrap, 572 and jackknife variance estimators for the $k$-Nearest Neighbors technique with illustrations 573 using forest inventory and satellite image data. Remote Sens. Environ. 115(12): 3165-3174. 574 doi: 10.1016/j.rse.2011.07.002. 
575 Næsset, E. 2004a. Accuracy of forest inventory using airborne laser scanning: evaluating the 576 first Nordic full-scale operational project. Scand. J. For. Res. 19(6): 554-557.

577 Næsset, E. 2004b. Practical large-scale forest stand inventory using a small-footprint airborne 578 scanning laser. Scand. J. For. Res. 19(2): 164-179.

579 Nieschulze, J., and Saborowski, J. 2002. Monitoring of Forests under Continuous Cover 580 System Management. In Continuous Cover Forestry: Assessment, Analysis, Scenarios.

581 Edited by K. von Gadow and J. Nagel and J. Saborowski. Springer Netherlands, Dordrecht. 582 pp. 53-66.

583 Nordman, D.J., and Caragea, P.C. 2008. Point and interval estimation of variogram models 584 using spatial empirical likelihood. J. Am. Stat. Assoc. 103(481): 350-361.

585 Opsomer, J.D., Breidt, F.J., Moisen, G.G., and Kauermann, G. 2007. Model-assisted 586 estimation of forest resources with generalized additive models. J. Am. Stat. Assoc.

587 102(478): 400-409.

588 Parker, R.C., and Evans, D.L. 2004. An application of LiDAR in a double-sample forest 589 inventory. W. J. Appl. For. 19(2): 95-101.

590 Rahlf, J., Breidenbach, J., Solberg, S., Næsset, E., and Astrup, R. 2014. Comparison of four 591 types of 3D data for timber volume estimation. Remote Sens. Environ. 155: 325-333. doi:

592 http://dx.doi.org/10.1016/j.rse.2014.08.036.

593 Rao, J.N., and Molina, I. 2015. Small area estimation. 2 ed. John Wiley \& Sons. pp. 480.

594 Salas, C., Ene, L., Gregoire, T.G., Næsset, E., and Gobakken, T. 2010. Modelling tree 595 diameter from airborne laser scanning derived variables: a comparison of spatial statistical 596 models. Remote Sens. Environ. 114(6): 1277-1285.

597 Salvati, N., Tzavidis, N., Pratesi, M., and Chambers, R. 2012. Small area estimation via M598 quantile geographically weighted regression. TEST 21(1): 1-28. doi: 10.1007/s11749-010599 0231-1.

600 Schreuder, H.T., Gregoire, T.G., and Wood, G.B. 1993. Sampling methods for multiresource 601 forest inventory. Wiley, New York. pp. 416.

602 Searle, S.R., Casella, G., and McCulloch, C.E. 1992. Variance components. Wiley, New 603 York. pp. 501.

604 Smith, H.F. 1938. An empirical law describing heterogeneity in the yields of agricultural 605 crops. J.Agric.Sci. 28: 1-23.

606 Smith, T.M., and Urban, D.L. 1988. Scale and resolution of forest structural pattern. Plant 607 Ecol. 74(2): 143-150.

608 Stein, A., and Ettema, C. 2003. An overview of spatial sampling procedures and experimental 609 design of spatial studies for ecosystem comparisons. Agric. Ecosyst. Environ. 94(1): 31-47.

610 Varma, V.K., Ferguson, I., and Wild, I. 2000. Decision support system for the sustainable 611 forest management. For. Ecol. Manage. 128: 49-55.

612 Ver Hoef, J.M., and Temesgen, H. 2013. A Comparison of the Spatial Linear Model to

613 Nearest Neighbor (k-NN) Methods for Forestry Applications. PLoS ONE 8(3): e59129. doi:

614 10.1371/journal.pone.0059129.

615 Wolfram, S. 2016. The Mathematica Documentation Center (Version 11.1). Wolfram

616 Research, Champaign, IL. p. 1470. 
617 Wulder, M., Coops, N., Hudak, A., Morsdorf, F., Nelson, R., Newnham, G., and Vastaranta, 618 M. 2013. Status and prospects for LiDAR remote sensing of forested ecosystems. Can. J.

619 Rem. Sens. 39(sup1): S1-S5.

620 Zhang, H. 2004. Inconsistent Estimation and Asymptotically Equal Interpolations in Model621 Based Geostatistics. J. Am. Stat. Assoc. 99(465): 250-261. doi:

$62210.1198 / 016214504000000241$.

623 Zhang, L., Gove, J.H., and Heath, L.S. 2005. Spatial residual analysis of six modeling 624 techniques. Ecol. Model. 186(2): 154-177. doi:

625 http://dx.doi.org/10.1016/j.ecolmodel.2005.01.007.

626 Zimmerman, D.L. 2006. Optimal network design for spatial prediction, covariance parameter 627 estimation, and empirical prediction. Environmetrics 17(6): 635-652.

628

629 
630 Table 1. Notation.

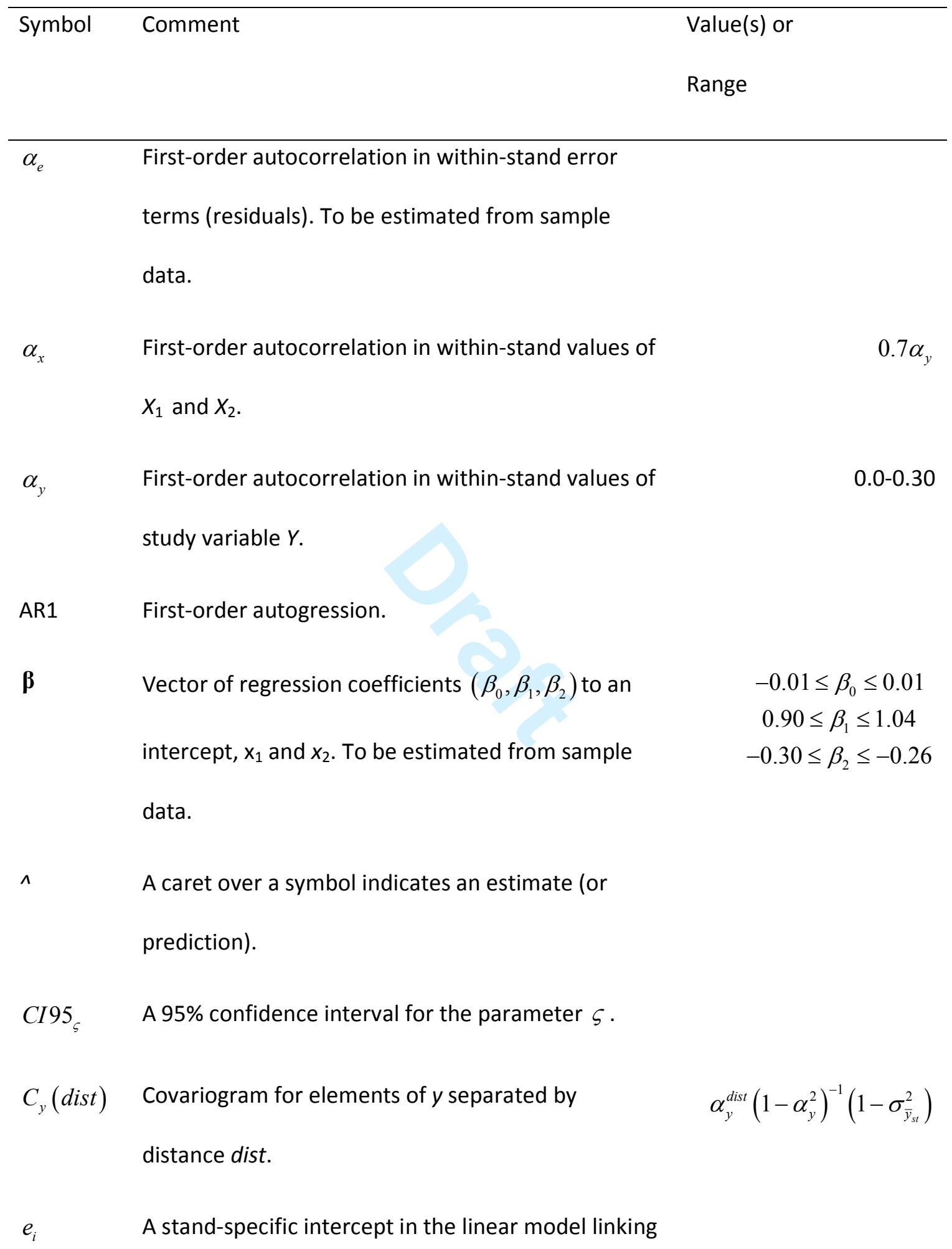


$X$ to $Y, i=1, \ldots, M$.

$e_{i j} \quad$ A stand and element specific error term in the linear

model linking $\mathbf{X}$ to $Y$.

$F_{k, l} \quad$ F-ratio random variable with $k$ and $/$ degrees of

freedom

$H_{0} \quad$ The null hypothesis

M Number of forest stands in a population.

$m_{i} \quad$ Number of elements in a stand.

25-196

$n \quad$ Sample size in number of fixed area plots

$120-480$ (elements).

$n_{1} \quad$ Number of sampled forest stands.

$n_{2} \quad$ Number of fixed area plots (elements) sampled

within each of $n_{1}$ sampled forest stands.

$N \quad$ Population size in number of elements (known).

$\Omega \quad 3 \times 3$ covariance matrix for coefficients in $\boldsymbol{\beta}$.

$P\left(\hat{\theta} \mid H_{0}\right)$ The probability that the random variable $\theta$ is equal to $\hat{\theta}$ or greater under the null hypothesis.

PSU Primary sampling unit (a fixed area element viz. a sample plot).

R A matrix of correlation coefficients. 
$R^{2} \quad$ Coefficient of determination in regression of $Y$ on $\mathbf{X}$.

$\rho^{2}\left(y, x_{1}\right) \quad$ Squared correlation coefficient between $y$ and $x_{1}$.

$\rho\left(y, x_{2}\right) \quad$ Correlation coefficient between $y$ and $x_{2}$.

$\rho\left(x_{1}, x_{2}\right) \quad$ Correlation coefficient between $x_{1}$ and $x_{2}$.

$\sigma_{s t}^{2} \quad$ The among-stand variance of $e_{i}$.

$\sigma_{w}^{2} \quad$ The within-stand variance of $e_{i j}$.

$\sigma_{\bar{y}_{s t}}^{2} \quad$ The among-stand variance of stand means of $Y$.

$\sigma_{\bar{x}_{s t}}^{2} \quad$ The among-stand variance of stand means of $X_{1}$ and $x_{2}$

$\Sigma \quad$ A variance-covariance matrix.

$T_{c o} \quad$ Total within-stand covariance.

$V(\varsigma) \quad$ The variance of a parameter $\varsigma$.

$\mathbf{X}$

A matrix of known element-level auxiliary variables

In this study $\mathbf{X}=\left(X_{1}, X_{2}\right)$. No specific attributes are

associated with $\mathbf{X}$. Variables $X_{1}$ and $X_{2}$ are assumed

normally distributed with a mean of zero and a

(total) variance of 1.0. $\approx 1.0833 \rho^{2}\left(y, x_{1}\right)$

$0.6,0.7$, and 0.8

$0.25 \rho\left(y, x_{1}\right)$

0.5

$\sigma_{\bar{y}_{s t}}^{2}\left(1-1.0833 \rho^{2}\left(y, x_{1}\right)\right)$

$\left(1-R^{2}\right)\left(1-\sigma_{\bar{y}_{s t}}^{2}\right)$

$0.05,0.10$, and 0.20

$0.7 \sigma_{\bar{y}_{s t}}^{2}$

$\mathbf{X}^{t} \quad$ The transpose of $\mathbf{X}$.

$Y \quad$ A generic (unspecified) normally distributed 
element-level study variable assumed normally distributed with a mean of zero and a variance of

1.0.

$y$

A realized value of $Y$. Values of $y$ are simulated for all elements in a population but only $y$ in elements in a sample, are known to an analyst.

$z$ A standard Gaussian variable (mean zero, variance 1.0).

631

632 


\section{Appendix}

634 Simulated populations

635

636 All populations used in this study are tri-variate $\left(y, x_{1}\right.$, and $\left.x_{2}\right)$ standard Gaussian populations

637 (mean zero, variance 1). Each population is composed of $M=800$ spatially compact forest

638 stands with $m_{\mathrm{i}}(i=1, \ldots, M)$ square fixed area elements arranged in a regular array. The value

639 of $m_{\mathrm{i}}$ was determined by a random draw from the set $\left\{5^{2}, 6^{2}, \ldots, 14^{2}\right\}$. Consequently, the

640 expected number of elements in a population $(N)$ is 97733 with a standard deviation of 2072.

641 The 36 populations vary with respect to the relative strength of stand effects in $Y$ and $\mathbf{X}$, a tri-

642 variate correlation structure, and a first-order autoregression in element values of $Y$ and $\mathbf{X}$.

643 For the 9 populations with no first-order autoregression (i.e. $\alpha_{y}=0$ ) in $Y$ or $\mathbf{X}$, the values of

$644 X_{1}, X_{2}$, and $Y$ for a single stand were generated in a two-step process. In step one, $m_{i}$ within-

645 stand values were generated from a zero-centred tri-variate multivariate Gaussian distribution

646 with a variances and correlation structure as specified by a population-level defined tri-

647 variate correlation structure and within-stand variances (i.e. one minus the among-stand

648 variances in $Y$ and $\mathbf{X}$ ). The $m_{i}$ simulated triplets were then mean-shifted to a mean of zero. In

649 a second step, a stand-level effect was added by a single random draw from a zero-centred

650 tri-variate multivariate Gaussian distribution with variances $\sigma_{\bar{y}_{s t}}^{2}$ for $Y$, and $0.7 \sigma_{\bar{y}_{s t}}^{2}$ for $X_{1}$ and

$651 X_{2}$, and otherwise the same correlation structure as used for the simulation of within-stand

652 data. Note, the two error terms $e_{i}$ and $e_{i j}$ in (1) are not simulated explicitly; they remain

653 unknown except in a full census of the population.

654 For the 27 populations with a positive first-order distance dependent autoregressive process

655 in $Y$ and $\mathbf{X}$, the within-stand values of $X_{1}, X_{2}$, and $Y$ were generated through a different two-

656 step process following the principles of linear co-regionalization (Chilès and Delfiner 1999, 
657 ch. 5.6.5; Gneiting et al. 2010). In step one $m_{i}$ within-stand values of $X_{1}, X_{2}$ and $Y$, were 658 generated from a zero-centred tri-variate multivariate Gaussian matrix distribution (Gupta 659 and Nagar 1999) with row- and column covariance structures specified by population-level 660 defined tri-variate correlation structures and the first-order autoregression along rows and 661 columns. A Gaussian matrix distribution of a $\sqrt{m_{i}} \times \sqrt{m_{i}}$ random (univariate) matrix $\mathbf{z}$ is the 662 distribution of $\boldsymbol{\mu}_{z}+\Sigma_{\text {row }}^{0.5} \mathbf{z} \Sigma_{\text {col }}^{0.5}$ where $\boldsymbol{\mu}_{z}$ is the expectation of $\mathbf{z}$ (here the null matrix $\mathbf{0}$ ) and $663 \Sigma_{d i r}$, dir = row, col is the covariance matrix of the $\sqrt{m_{i}}$ elements in a row (column) of the 664 square matrix with $m_{\mathrm{i}}$ elements. Details on a matrix raised to a power of 0.5 are in Johnson et 665 al. (2001). Afterwards, the $m_{i}$ simulated triplets were mean-shifted to a mean of zero and 666 combined to a $m_{i} \times 3$ matrix and post-multiplied by $\mathbf{R}^{0.5}$ where $\mathbf{R}$ is the $3 \times 3$ the correlation 667 matrix of the three variables. In the second step, as before, stand-level effects were added by 668 a single random draw from a zero-centred tri-variate multivariate Gaussian distribution with 669 variances $\sigma_{\bar{y}_{s t}}^{2}$ for $Y$, and $0.7 \sigma_{\bar{y}_{s t}}^{2}$ for $X_{1}$ and $X_{2}$, and otherwise the same correlation structure as 670 used for the within-stand data. This procedure was implemented in the Mathematica ${ }^{\circledR} 11.0 .1$ 671 software (Wolfram 2016). A validation of the within-stand autoregression in $Y$ and $\mathbf{X}$ was 672 obtained by ordering the $m_{\mathrm{i}}$ elements to the shortest traverse through all elements in a stand 673 followed by a maximum likelihood estimation of $\alpha_{y}$ and $\alpha_{x_{k}}, k=1,2$ (Christensen 2001, p.

674 198). Two sets of estimates were computed and averaged; one by beginning the traverse from 675 the upper left corner of a stand, and a second by starting it from the lower right corner. We 676 learned that for a single stand the apparent realized first-order autoregression coefficient 677 could be up to $50 \%$ from its nominal value, but the average over 800 stands was typically 678 within $5 \%$ of a target, and the average over 800 stands and 200 replications was, typically, 679 within $0.5 \%$ of a target. The largest deviations were consistently associated with the smallest 680 stands with 25 elements. 
681 The simulations generated 9 levels of the within-stand residual error $\sigma_{w}^{2}$. With standard

682 Gaussian data and the linear model in (1) we have $\sigma_{w}^{2}=\left(1-R^{2}\right)\left(1-\sigma_{\bar{y}_{s t}}^{2}\right)$ where $R^{2}$ is the

683 coefficient of variation in the regression of $Y$ on $X_{1}$ and $X_{2}$. In our set up it holds that

$684 R^{2} \cong 1.0833 \rho^{2}\left(y, x_{1}\right)$ where the error of approximation is $\leq 0.002$. Ignoring this error, we

685 obtain - after rounding to the nearest 0.01 - the following levels of $\sigma_{w}^{2}: 0.11,0.12,0.13$,

$6860.19,0.22,0.28,0.32,0.33$, each represented by 48 settings of $n(4), n_{2}(3)$, and $\alpha_{y}(4)$.

687 There is also 9 level of the among-stand residual error $\sigma_{s t}^{2}$. The nine levels can be obtained (to

688 within 0.001$)$ from $\sigma_{s t}^{2} \cong \sigma_{\bar{y}_{s t}}^{2}\left(1-1.0833 \rho^{2}\left(y, x_{1}\right)\right)$. Specifically, the levels are $0.007,0.012$,

$6890.013,0.018,0.024,0.027,0.035,0.048$, and 0.070 . It follows from the overall design that

690 there are only 9 unique combinations of $\sigma_{s t}^{2}$ and $\sigma_{w}^{2}$, each represented by 48 settings of $n, n_{2}$,

691 and $\alpha_{y}$.

692 Estimation of $\alpha$ from $\hat{y}_{i j}$

693 In our populations we introduced a first-order autoregressive process (AR1) in both $Y$ and $\mathbf{X}$

694 with parameters $\alpha_{y}=\{0.0,0.1,0.2,0.3\}$ and $\alpha_{x_{k}}=0.7 \alpha_{y}, k=1,2$. Since as per (1) we have

$695 y_{i j}=\boldsymbol{\beta}^{t} \mathbf{X}+e_{i}+e_{i j}$ it follows (Engel 1984, (7.1) in proposition 4) that for a known $\boldsymbol{\beta}$ and $e_{i}$

696 the prediction $\hat{y}_{i j}=\boldsymbol{\beta}^{t} \mathbf{X}+e_{i}$ must also follows an AR1 process for all admissible lags (along

697 rows or columns) of $k$ elements (here 1 to $\left(1\right.$ to $\left.\sqrt{m_{i}}\right)$. We can now state: for the elements

698 within a stand $e_{i j}$ is the difference between two AR1 processes which means that $e_{i j}$ also

699 follows an AR1 process (Engel 1984, (4.2) in proposition 1). The only solution to the AR1

700 parameters satisfying the constraint on the autocorrelation functions of the involved random

701 variables in (4.2) (Ibid.) requires $\alpha_{y}=\alpha_{\hat{y}}=\alpha_{e}$. Naturally, the equation only holds with a 
702 census estimate of the regression coefficients, but it suggests an opportunity to estimate $\alpha_{e}$ 703 from $\alpha_{\hat{y}}$. 
704 Figure 1. Estimates of the within-stand variance component in residual errors plotted against

705 their nominal target values. A one-to-one line, is added for orientation.

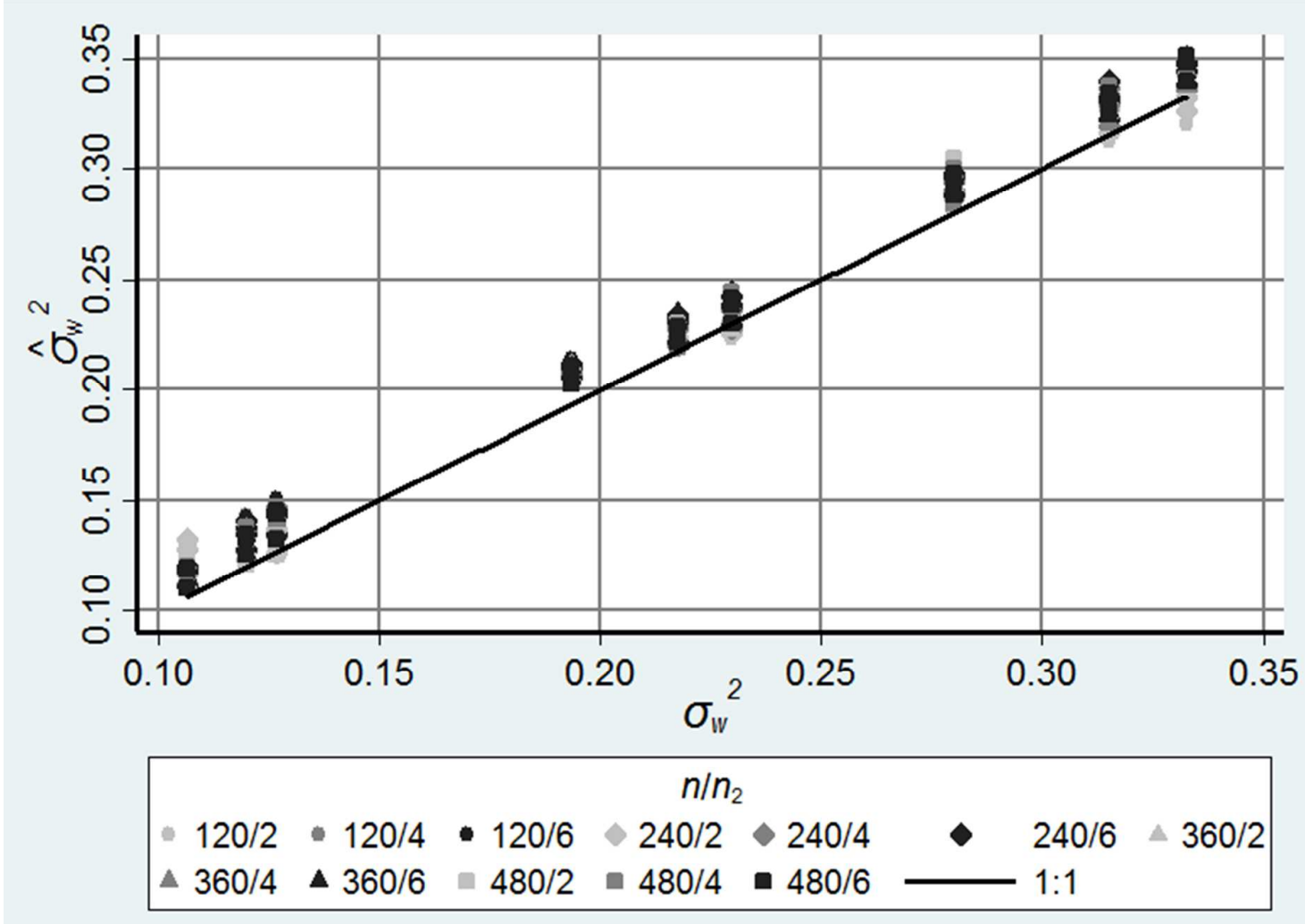

706

707 
708 Figure 2. Estimates of the among-stand variance component in residual errors plotted against 709 their nominal target value. A one-to-one line, is added for orientation.

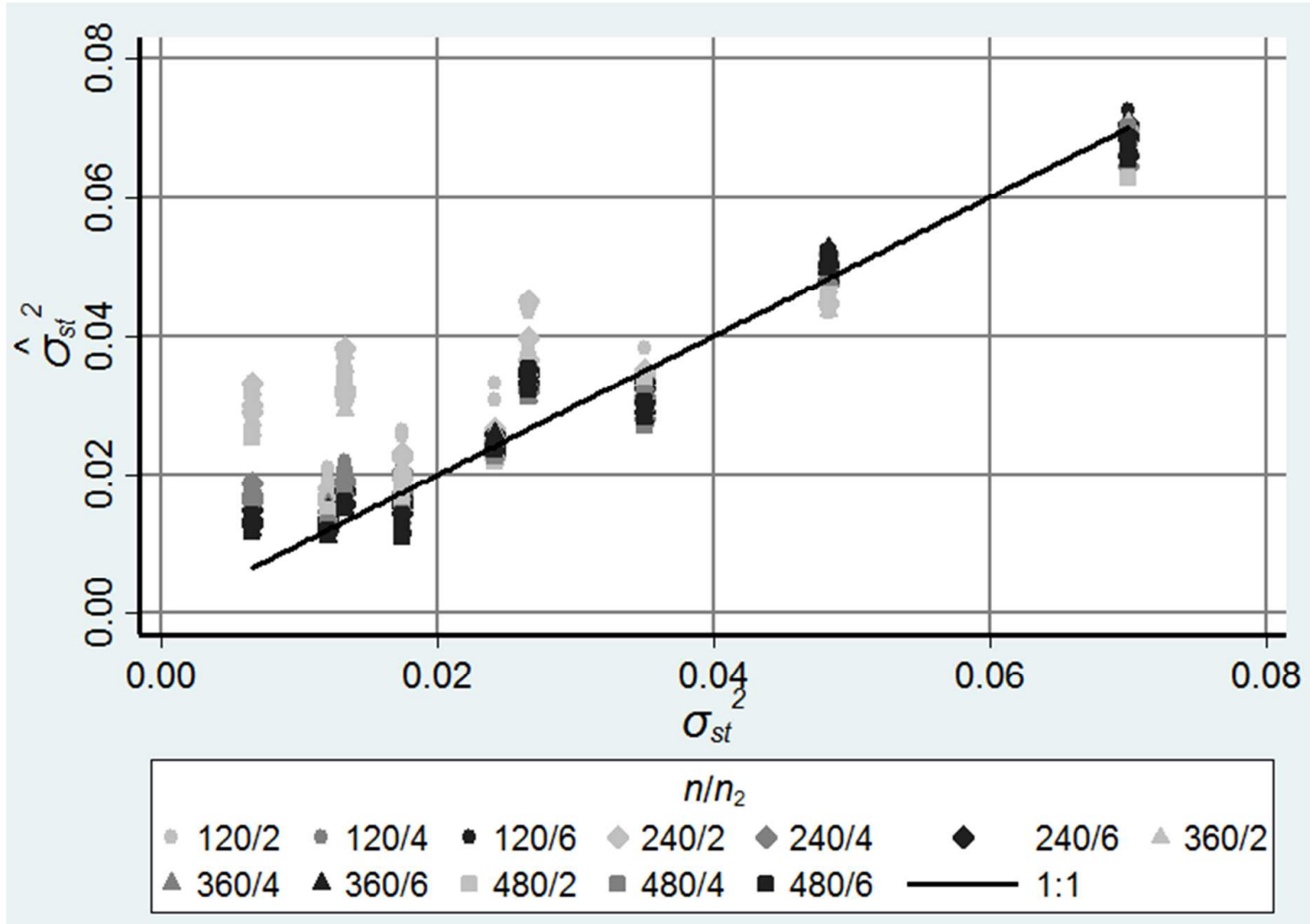

710

711 
712 Figure 3. Estimates of the first-order autoregressive coefficient $\alpha_{e}$ obtained from actual (true)

713 model residuals $\left(e_{i j}\right)$ plotted against the nominal first-order autoregressive coefficient $\alpha_{y}$ in

$714 y_{i j}$. A one-to-one line, is added for orientation.

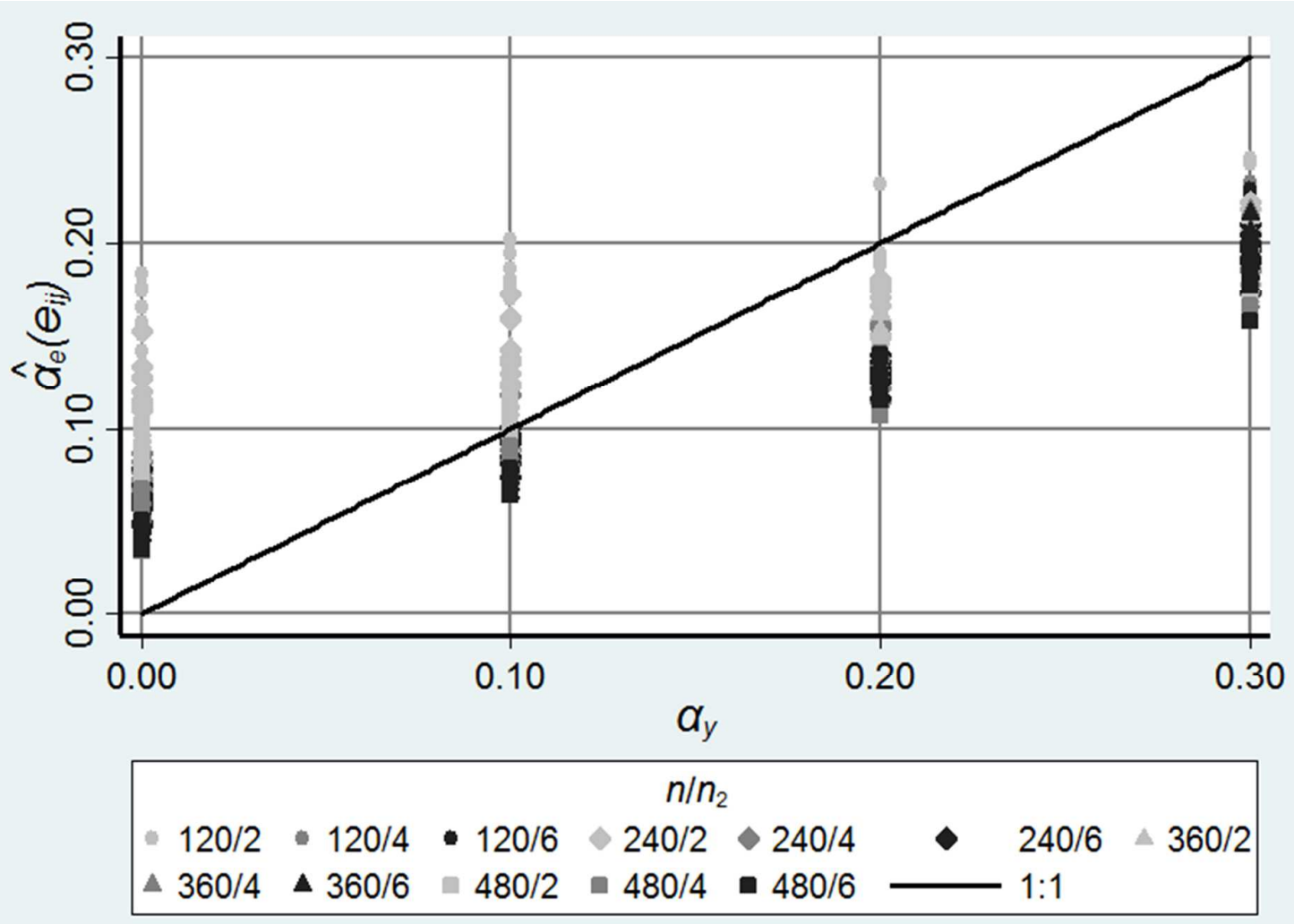

715

716 
717 Figure 4. Scatter plot of the first-order autoregressive coefficient $\alpha$ obtained from empirical

718 residuals $\left(\hat{e}_{i j}\right)$ plotted against estimates obtained from actual (true) residuals $\left(e_{i j}\right)$. A one-to-

719 one line, is added for orientation.

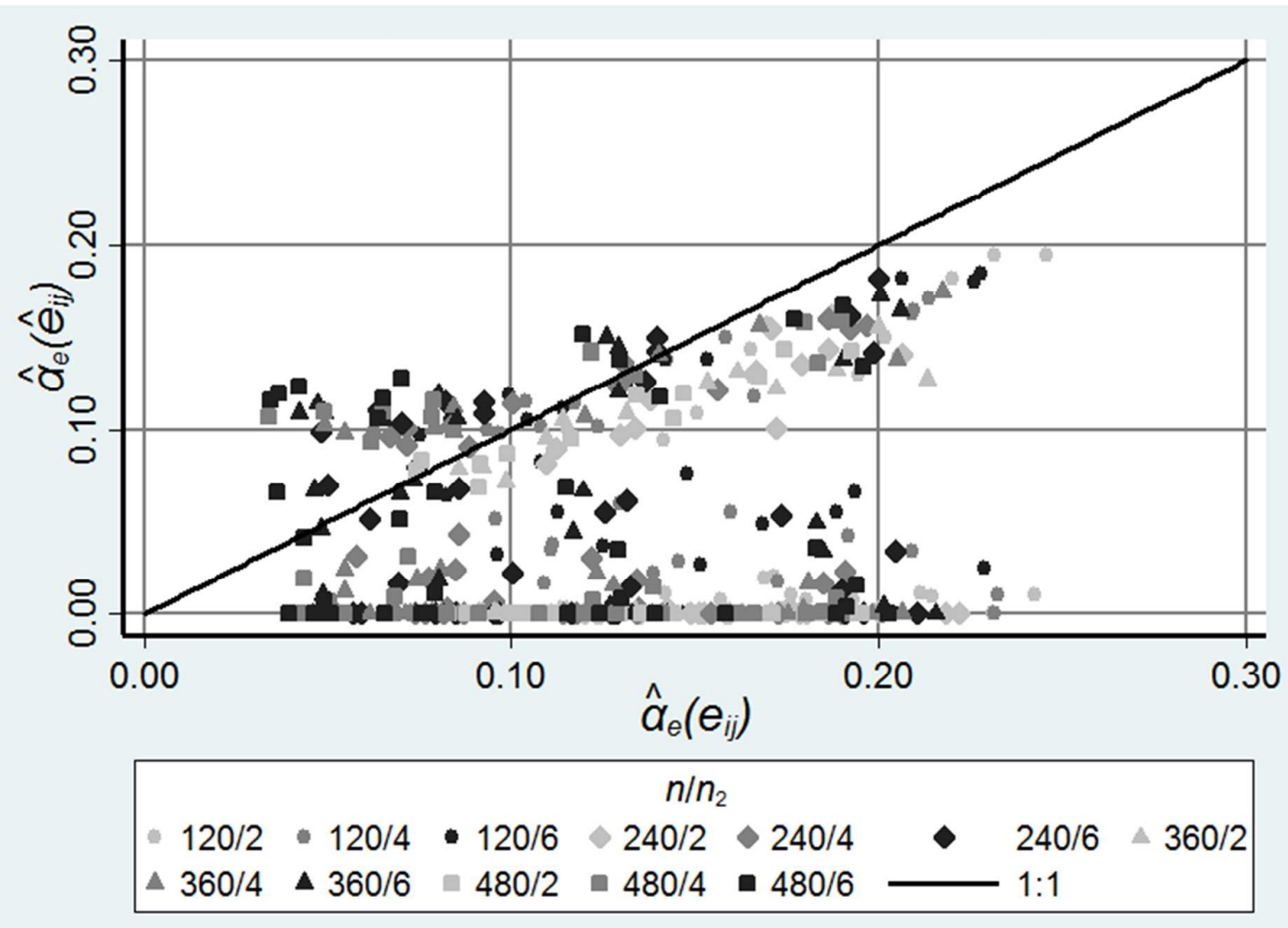


722 Figure 5. Estimates of the first-order autoregressive coefficient $\alpha_{e}$ obtained from model-based

723 expectations of $y_{i j}\left(\right.$ i.e. $\left.\hat{y}_{i j}\right)$ plotted against the nominal first-order autoregressive coefficient

$724 \alpha_{y}$ in $y_{i j}$. A one-to-one line, is added for orientation.

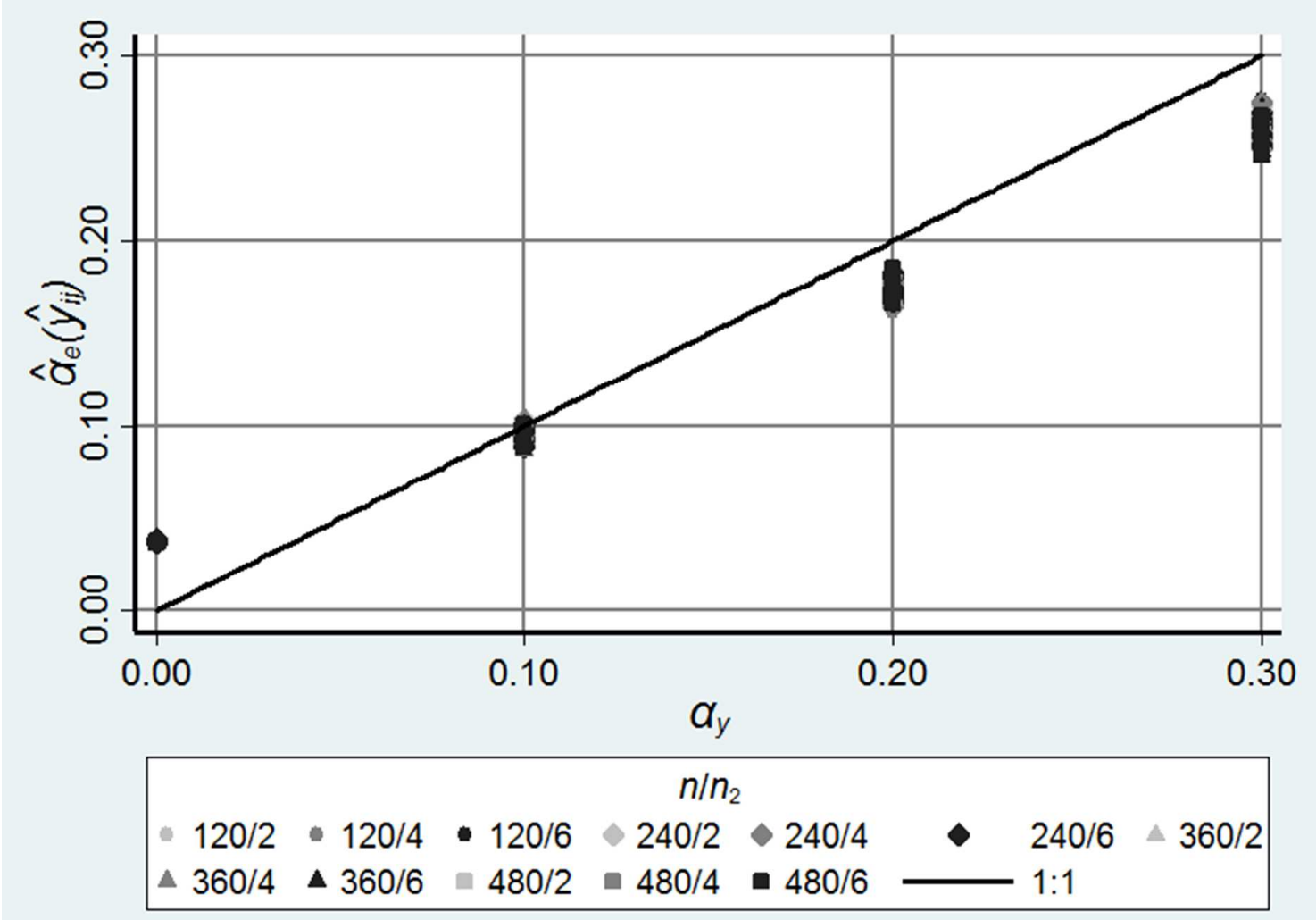

725

726 
727 Figure 6. Estimates of the variance of a synthetic stand mean (see (3)) plotted against the 728 actual mean squared error of the synthetic mean. Variances were obtained with $\alpha$ estimated 729 from $\hat{y}_{i j}$. A one-to-one line, is added for orientation.

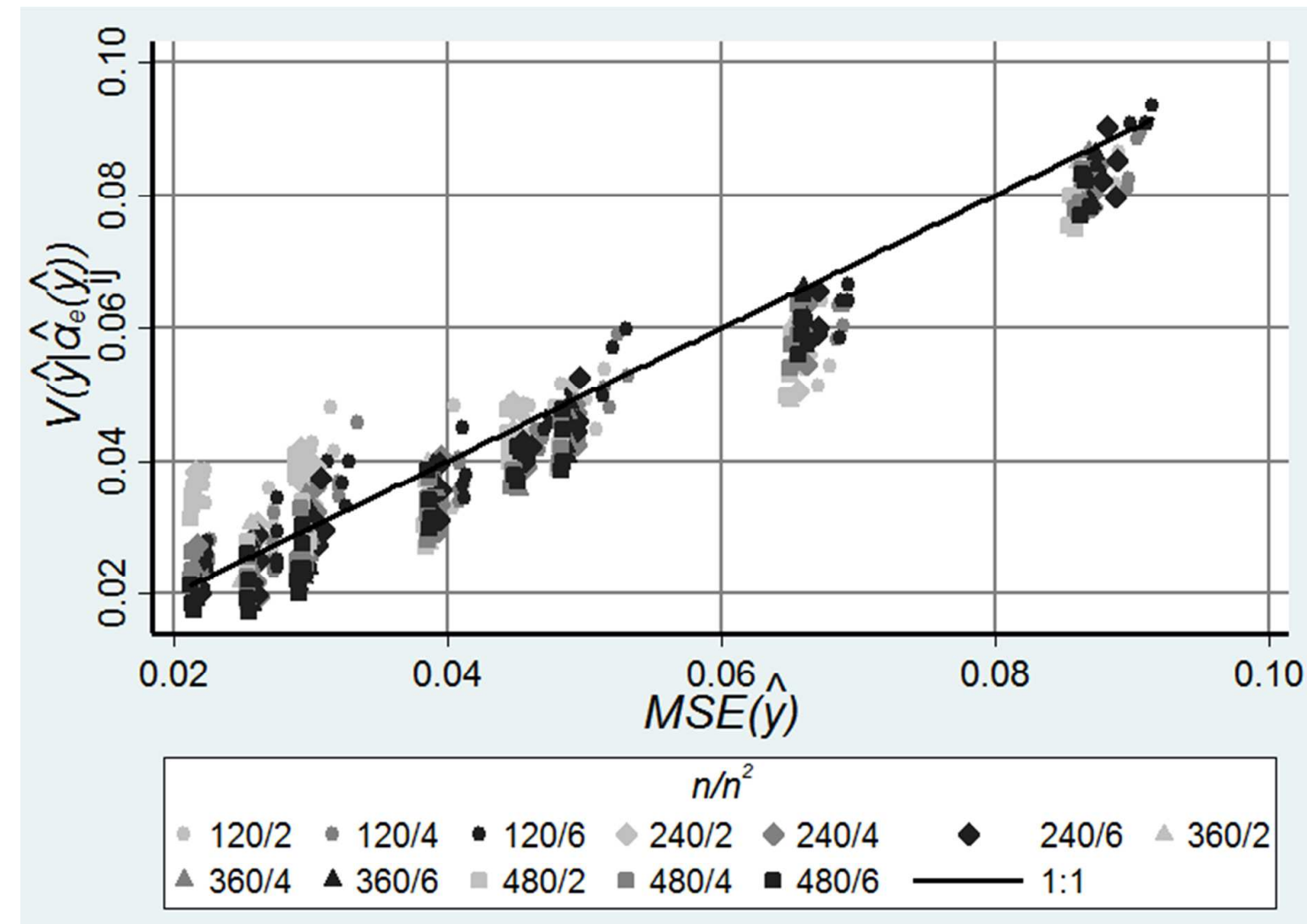


732 Figure 7. Estimates of the variance of a synthetic stand mean obtained with $\alpha_{e}$ estimated 733 from empirical residuals $\left(\hat{e}_{i j}\right)$ plotted against variances obtained with $\alpha_{e}$ estimated from 734 model-based expectations $\left(\hat{y}_{i j}\right)$. A one-to-one line, has been added for orientation.

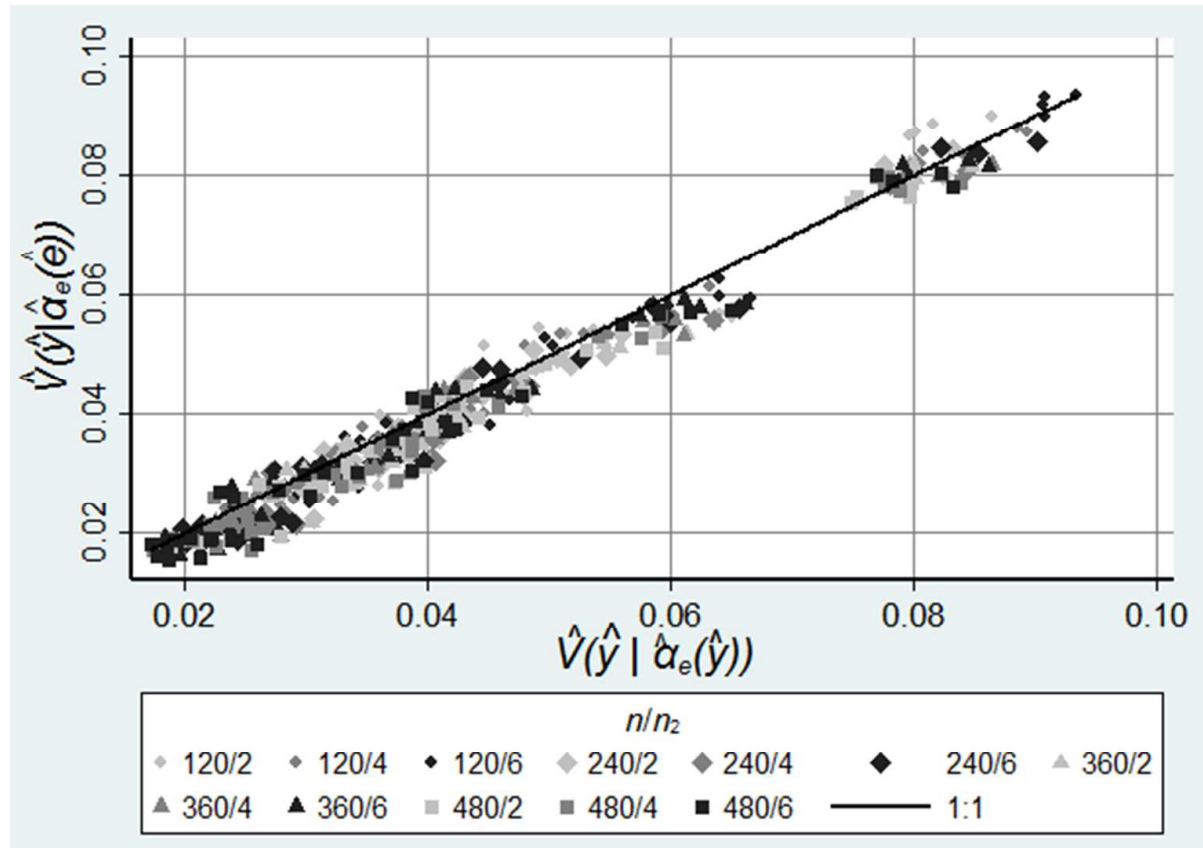


737 Figure 8. Achieved coverage ( $\hat{C} O$ ) of nominal CI95s plotted against nominal target values of 738 the among stand variance component of model residuals $\left(\sigma_{s t}^{2}\right)$. The target coverage of 0.95 is 739 highlighted (in bold).

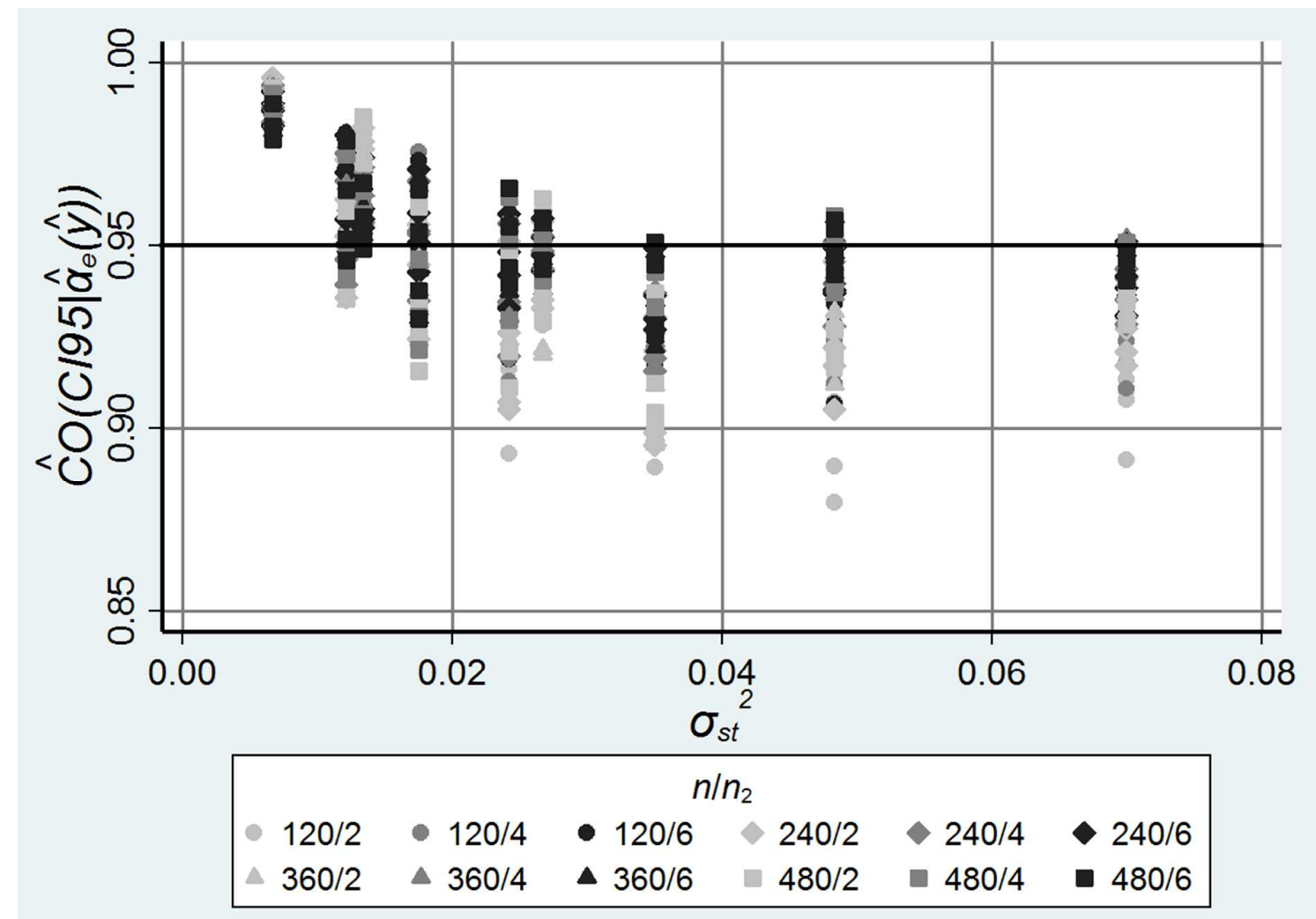

